Article

\title{
Probing the Influence of Linker Length and Flexibility in the Design and Synthesis of New Trehalase Inhibitors
}

\author{
Giampiero D'Adamio ${ }^{1}$, Matilde Forcella ${ }^{2}{ }^{(0)}$, Paola Fusi ${ }^{2}$, Paolo Parenti ${ }^{3}$, \\ Camilla Matassini ${ }^{1,4, *(D)}$, Xhenti Ferhati ${ }^{1}$, Costanza Vanni ${ }^{1}$ (D) and Francesca Cardona ${ }^{1,4,5, * \text { (D) }}$ \\ 1 Department of Chemistry 'Ugo Schiff', University of Florence, via della Lastruccia 3-13, \\ I-50019 Sesto Fiorentino (FI), Italy; giampiero_dadamio@hotmail.it (G.D.); mage90@hotmail.it (X.F.); \\ costanza.vanni@unifi.it (C.V.) \\ 2 Department of Biotechnology and Biosciences, University of Milano-Bicocca, Piazza della Scienza 2, \\ 20126 Milano, Italy; matilde.forcella@unimib.it (M.F.); paola.fusi@unimib.it (P.F.) \\ 3 Department of Earth and Environmental Sciences, University of Milano-Bicocca, Piazza della Scienza 1, \\ 20126 Milano, Italy; paolo.parenti@unimib.it \\ 4 Associated with CNR-INO, Via N. Carrara 1, I-50019 Sesto Fiorentino (FI), Italy \\ 5 Associated with Consorzio Interuniversitario Nazionale di ricerca in Metodologie e Processi Innovativi di \\ Sintesi (CINMPIS), Università di Bari, 70125 Bari, Italy. \\ * Correspondence: camilla.matassini@unifi.it (C.M.); francesca.cardona@unifi.it (F.C.); \\ Tel.: +39-055-457-3504 (F.C.)
}

Received: 30 January 2018; Accepted: 14 February 2018; Published: 16 February 2018

\begin{abstract}
This work aims to synthesize new trehalase inhibitors selective towards the insect trehalase versus the porcine trehalase, in view of their application as potentially non-toxic insecticides and fungicides. The synthesis of a new pseudodisaccharide mimetic $\mathbf{8}$, by means of a stereoselective $\alpha$-glucosylation of the key pyrrolizidine intermediate 13, was accomplished. The activity of compound 8 as trehalase inhibitor towards C. riparius trehalase was evaluated and the results showed that 8 was active in the $\mu \mathrm{M}$ range and showed a good selectivity towards the insect trehalase. To reduce the overall number of synthetic steps, simpler and more flexible disaccharide mimetics $\mathbf{9 - 1 1}$ bearing a pyrrolidine nucleus instead of the pyrrolizidine core were synthesized. The biological data showed the key role of the linker chain's length in inducing inhibitory properties, since only compounds 9 $(\alpha, \beta$-mixture), bearing a two-carbon atom linker chain, maintained activity as trehalase inhibitors. A proper change in the glucosyl donor-protecting groups allowed the stereoselective synthesis of the $\beta$-glucoside $9 \beta$, which was active in the low micromolar range $\left(\mathrm{IC}_{50}=0.78 \mu \mathrm{M}\right)$ and 12 -fold more potent (and more selective) than $9 \alpha$ towards the insect trehalase.
\end{abstract}

Keywords: iminosugars; trehalase inhibitors; pseudodisaccharides; pyrrolizidines; pyrrolidines; glycosylation reaction

\section{Introduction}

Trehalose ( $\alpha$-D-glucopyranosyl $\alpha$-D-glucopyranoside, 1, Figure 1) is a disaccharide with a multifunctional physiological role in various organisms (e.g., bacteria, fungi, invertebrates and higher plants) [1]. The role of trehalose in insects is of particular importance connected to its hydrolysis operated by the enzyme $\alpha$-trehalase (EC 3.2.1.28). Indeed, $\alpha$-trehalase is an inverting glycosidase [2] that promotes the conversion of trehalose into two molecules of glucose, which is vital for insect flight and essential for larvae resistance to stress factors. While trehalose is not present in mammalian cells, humans do possess the enzyme trehalase, only at intestinal level, probably to hydrolyze occasionally ingested trehalose. Indeed, intolerance to fungi has been correlated with the absence or deficit of 
trehalases in mammals [1]. For the important role of trehalose-derived glucose in larvae survival and development, trehalase inhibitors have been considered in recent years an interesting target for the identification of novel insecticides and fungicides. However, due to the presence of trehalase also in mammals, specificity towards insect trehalase is crucial for the development of drugs that are safe for plants and mammals, and possibly also for insects that are of benefit in nature [3].

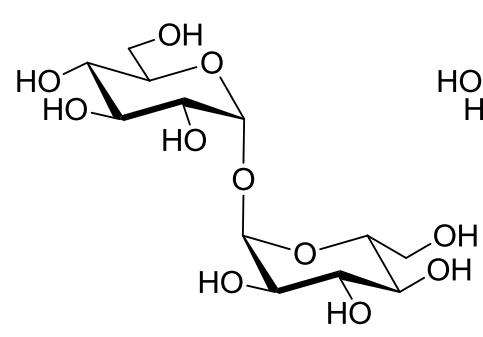

trehalose (1)

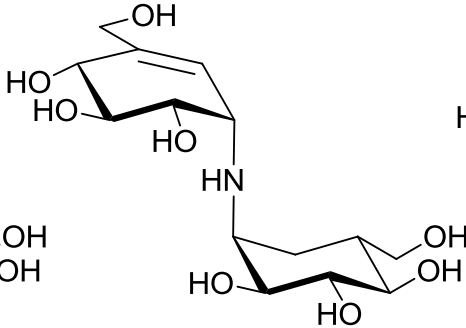

validoxylamine $A(2)$

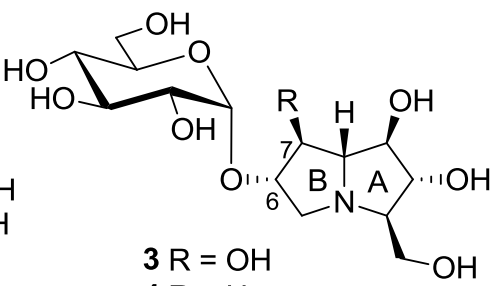

$4 \mathrm{R}=\mathrm{H}$

$5 \mathrm{R}=\mathrm{CH}_{2} \mathrm{OH}$

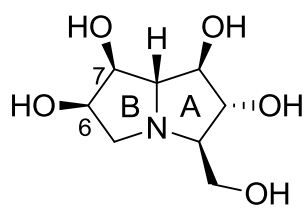

(-)-Uniflorine A (6)

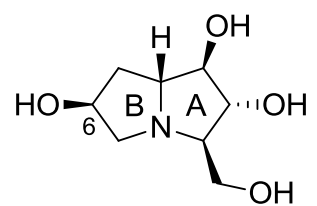

7-deoxy-uniflorine A (7)

Figure 1. Trehalose (1), the natural substrate of the enzyme $\alpha$-trehalase (EC 3.2.1.28), and some natural and non-natural trehalase inhibitors reported to date.

Among the most powerful inhibitors of trehalases there are some natural pseudodisaccharides and analogs shown in Figure 1, such as validoxylamine (2), casuarine-6-O-D-glucoside 3 and its non-natural analogues 4 and 5 . The first investigated trehalase three-dimensional structure was the family 37 periplasmic enzyme from E. coli (Tre37A), which was solved in complex with 2 [4], with casuarine-6-O-D-glucoside 3 [5] and with non-natural analogue 5 [6]. Our findings showed that, with $E$. coli trehalase, casuarine-based inhibitors are placed within the primary catalytic site with the A ring of the pyrrolizidine nucleus that mimics the natural glucose configuration $[5,6]$. However, subtle changes at ring B (e.g., modification at C-7 as in compound 5) were able to confer both potency and specificity in trehalase inhibition [6]. More interestingly, we later found that simpler pseudomonosaccharide inhibitors such as natural (-)-uniflorine A (6) and non-natural analogue 7-deoxy-uniflorine A (7) showed an excellent inhibitory profile, being completely selective towards the insect trehalase, although less potent in absolute value with respect to casuarine-6-O-D-glucoside derivatives 3-5 [7]. It is worth noting that compounds 6 and 7 bear the opposite configuration at C-6 in ring B with respect to the previously investigated casuarine-6-O-D-glucoside and analogues (compounds 3-5).

Following our interest in the identification of new more potent and selective trehalase inhibitors, we envisaged that the synthesis of a pseudodisaccharide 8 (7-deoxyuniflorine A glucoside), analogue of disaccharide 4 but with an opposite configuration at C-6, would furnish a promising inhibitor (Figure 2). A straightforward approach to compound 8 is reported in this work.

In consideration of the high number of steps required for the synthesis of pseudodisaccharides bearing the pyrrolizidine nucleus such as compounds 3-5 and $\mathbf{8}$ itself, we also wanted to explore the insect trehalase activity of simpler pseudodisaccharide inhibitors 9-11, that bear the glucose unit linked to the pyrrolidine DAB-1 instead of the pyrrolizidine nucleus. DAB-1 actually has the same structure of ring A of the pyrrolizidine nucleus (Figure 2), and we had previously verified that it is able to mimic glucose by imparting trehalase inhibition in pseudodisaccharide mimetics $[8,9]$. The aim of the present work was to link the DAB-1 moiety to the glucose moiety through a linker of variable 
length (2, 3 and 4 carbon atoms), in order to verify which was the more appropriate tether length (Figure 2) for trehalase inhibition.

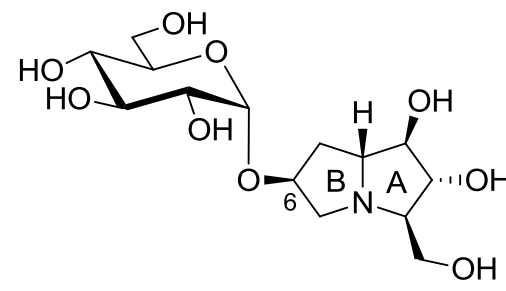

7-deoxyuniflorine A glucoside (8)

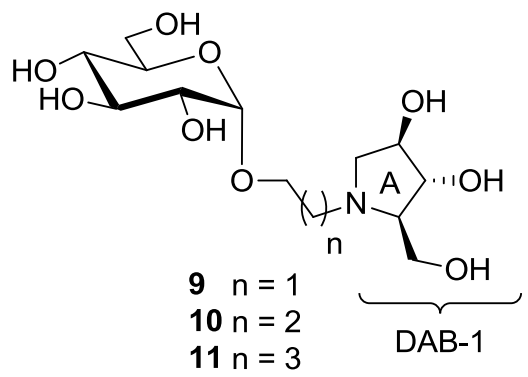

$10 \mathrm{n}=2$

$11 n=3$

Figure 2. This work: synthesis of a new pseudodisaccharide mimetic 8 and of a series of more flexible disaccharide mimics $\mathbf{9 - 1 1}$ bearing the DAB-1 nucleus instead of the pyrrolizidine moiety.

The synthesis of both pyrrolizidine-based (8) and pyrrolidine-based (9-11) pseudodisaccharides involved a common key intermediate: the enantiopure nitrone $\mathbf{1 2}$, readily available from D-arabinose in 5 steps (Scheme 1) [10]. However, while on one hand 5 further steps are necessary to access the pyrrolizidine glucosyl acceptor (lactam 13) [7], the hydroxypyrrolidine glucosyl acceptors 15-17 are in principle readily available from amine 14 [11,12]. Pyrrolidine 14, recently synthesized by some of us in a one-pot 2-steps sequence from nitrone 12 (Scheme 1) [13], has the same stereochemical pattern of the hydroxyl groups of ring A in lactam 13, which is responsible for the key interactions within the enzyme active site of the final compounds [5,6]. Therefore, compound $\mathbf{1 4}$ is in principle able to mimic the more complex pyrrolizidine skeleton of lactam 13. We also reasoned that pyrrolidine 14 could be easily functionalized at the endocyclic nitrogen atom to afford the desired glucosyl acceptors 15-17, allowing to probe different spatial lengths between the sugar and the iminosugar unit of the target pseudodisaccharides.

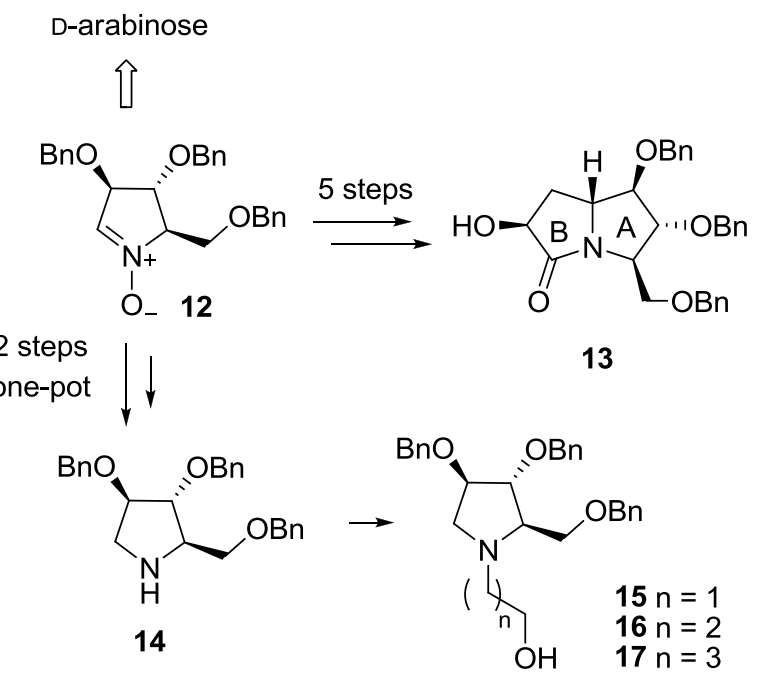

Scheme 1. Synthetic steps to access pyrrolizidine lactam $\mathbf{1 3}$ and hydroxypyrrolidines 15-17 (through the key intermediate 14) from nitrone $\mathbf{1 2 .}$

This work has therefore a dual aim: from one side to investigate the synthesis and biological activity of a new and differently configured pseudodisaccharide mimic (compound 8), and from the other side to explore the possibility to obtain simpler and more flexible inhibitors (compounds 9-11). The results in this direction are shown herein, and highlight the crucial role of the linker length in the design of more flexible inhibitors. 


\section{Results and Discussion}

The synthesis of the new disaccharide mimetic 8 started from lactam 13, whose preparation was recently reported by some of us [7]. Compound 13 bears a free $\mathrm{OH}$ group at C-6, and was therefore employed as the acceptor in the glucosylation reaction with the benzyl protected glucopyranosyl trichloroacetimidate 18 (Scheme 2) [14]. The reaction, performed in diethyl ether at $-20{ }^{\circ} \mathrm{C}$, was highly selective in favor of the $\alpha$-anomer, as reported before for the casuarine derivatives [5,6], and afforded the $\alpha$ glucoside $19 \alpha$ as the major product ( $86 \%$ yield). Small amounts ( $4 \%$ yield) of the $\beta$-anomer $19 \beta$ were isolated and characterized. Reduction of the $\mathrm{C}=\mathrm{O}$ double bond in $19 \alpha$ with $\mathrm{LiAlH}_{4}$ in THF at reflux temperature afforded compound 20 in excellent yield (90\%). Catalytic hydrogenation, followed by treatment with the ion exchange resin 50WX8-200 gave 7-deoxy-uniflorine A glucoside 8 in $93 \%$ yield over two steps (Scheme 2). The ${ }^{1} \mathrm{H}-\mathrm{NMR}$ spectrum of compound 8 showed a $d d$ at 3.30 ppm for H-2 signal, with coupling constants of 9.8 and $3.5 \mathrm{~Hz}$, respectively. This indicates an axial-axial relationship with $\mathrm{H}-3$ and an axial-equatorial relationship with $\beta \mathrm{H}-1$, and therefore confirms the $\alpha$-configuration of the glucose moiety.<smiles>O=C1[C@H](O)C[C@H]2[C@@H](OCc3ccccc3)[C@H](OCc3ccccc3)C(COCc3ccccc3)N12</smiles>

13

$90 \%$

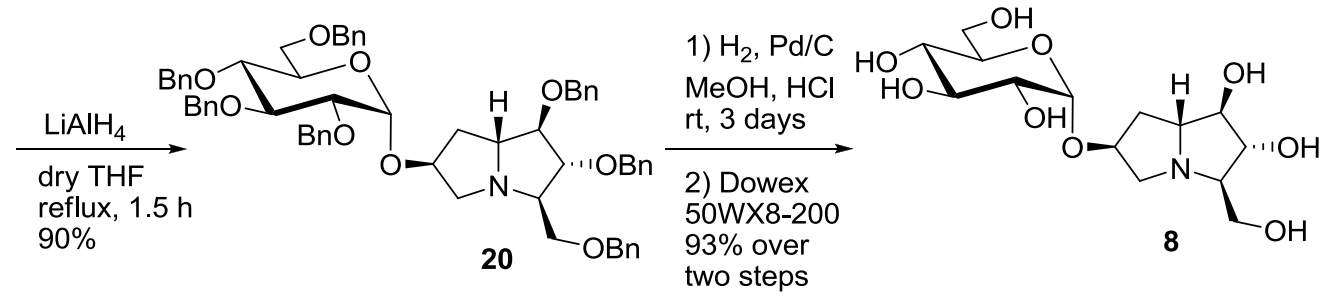

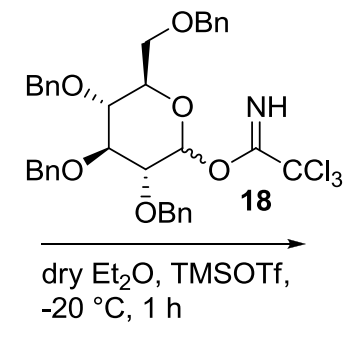

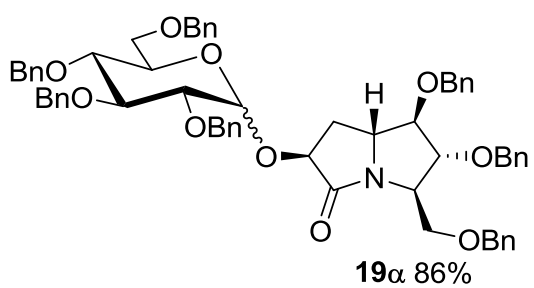

$19 \alpha 86 \%$
$19 \beta 4 \%$

Scheme 2. Synthesis of the pseudodisaccharide 7-deoxyuniflorine A glucoside (8).

In order to reduce the overall number of synthetic steps necessary to access the glucosyl acceptor in the final glucosylation with trichloroacetimidate 18, we also designed and prepared a series of pseudodisaccharide derivatives 9-11 (Scheme 1) containing a DAB-1 nucleus and a remaining D-glucose unit linked through a 2, 3 or 4-carbon atoms spacer.

Pyrrolidine 14 was $N$-alkylated with an excess of 2-bromo-1-ethanol, 3-bromo-1-propanol and 4-bromo-1-butanol in THF, using $\mathrm{Et}_{3} \mathrm{~N}$ as the base at room temperature, affording alcohols 15 (85\% yield) [12], 16 (94\% yield) and 17 (88\% yield), with a free hydroxyl group at the end of the aliphatic chain, thus allowing selective glucosylation at this position (Scheme 3 ).

The reaction of alcohols 15-17 with trichloroacetimidate donor 18 [14] in dry diethyl ether in the presence of trimethylsilyl trifluoromethanesulfonate (TMSOTf) gave 21 (50\% yield), 22 (36\%) and $23(66 \%)$ as $\alpha, \beta$ glucoside mixtures, impossible to be separated by flash column chromatography (Scheme 3). In all cases it was not possible to determine the $\alpha: \beta$ ratios by ${ }^{1} \mathrm{H}-\mathrm{NMR}$ analysis because the signals of the anomeric protons of the two anomers were covered by the $\mathrm{CH}_{2}$ benzyl groups signals. The glucosylation reactions were also performed in dry $\mathrm{CH}_{2} \mathrm{Cl}_{2}$ affording similar yields $(\mathbf{2 1}, \mathbf{7 6} \%, \mathbf{2 2}$, $39 \%$ and $23,50 \%$ ) of the $\alpha, \beta$ glucoside mixtures.

Catalytic hydrogenation of 21-23 $\alpha, \beta$ in acidic $\mathrm{MeOH}$ with $\mathrm{Pd} / \mathrm{C}$ gave the corresponding mixture of deprotected $\alpha, \beta$ isomers $\mathbf{9 - 1 1}$, as hydrochloride salts, that were passed onto an ion exchange resin (Dowex 50WX8-200) eluting successively with $\mathrm{MeOH}, \mathrm{H}_{2} \mathrm{O}$ and $6 \%$ aqueous ammonia. The final 
elution with ammonia afforded pure glucosyl derivatives 9-11 as a mixture of $\alpha$ and $\beta$-anomers, in $32-40 \%$ yields $\left(9 \alpha, \beta, \alpha: \beta 2: 1 ; 10 \alpha, \beta, \alpha: \beta 1: 1 ; 11 \alpha, \beta, \alpha: \beta 1.2: 1\right.$ as determined by ${ }^{1} \mathrm{H}$ NMR analysis, see Supplementary Materials).<smiles>BrC(Br)(Br)COC1CNCC1OCc1ccccc1</smiles>

14<smiles>c1ccc(CCOc2ccccc2)cc1</smiles>

$21 \alpha, \beta(n=1)$

$22 \alpha, \beta(\mathrm{n}=2)$

$23 \alpha, \beta(n=3)$

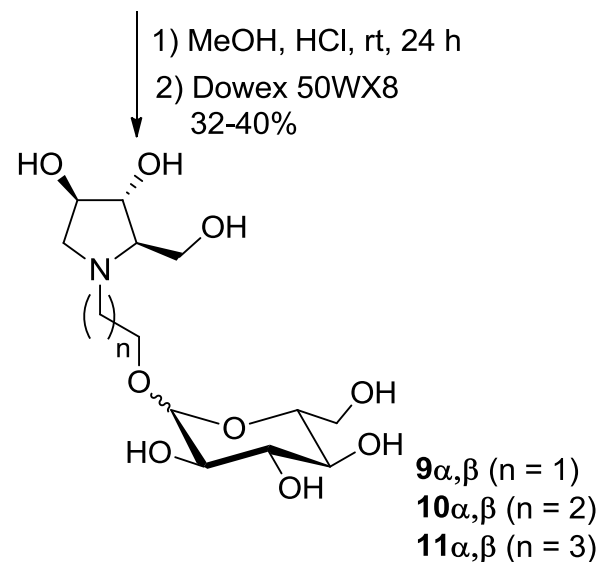

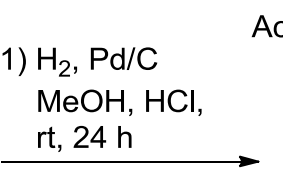

2) $\mathrm{Ac}_{2} \mathrm{O}, \mathrm{Py}$ $\mathrm{rt}, 16 \mathrm{~h}$

$$
11 \alpha, \beta(n=3)
$$

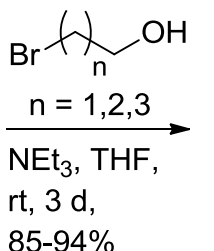<smiles>BrC(Br)(Br)c1ccccc1</smiles>

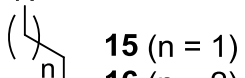

$n 16(n=2)$ $\mathrm{H}_{17}(n=3)$

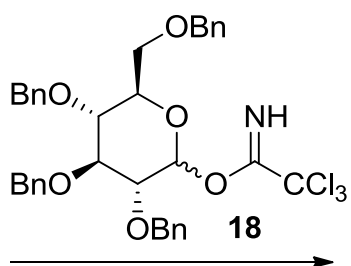

dry $\mathrm{Et}_{2} \mathrm{O}$ or $\mathrm{CH}_{2} \mathrm{Cl}_{2}$ TMSOTf, $-20{ }^{\circ} \mathrm{C}, 1 \mathrm{~h}$ $36-76 \%$

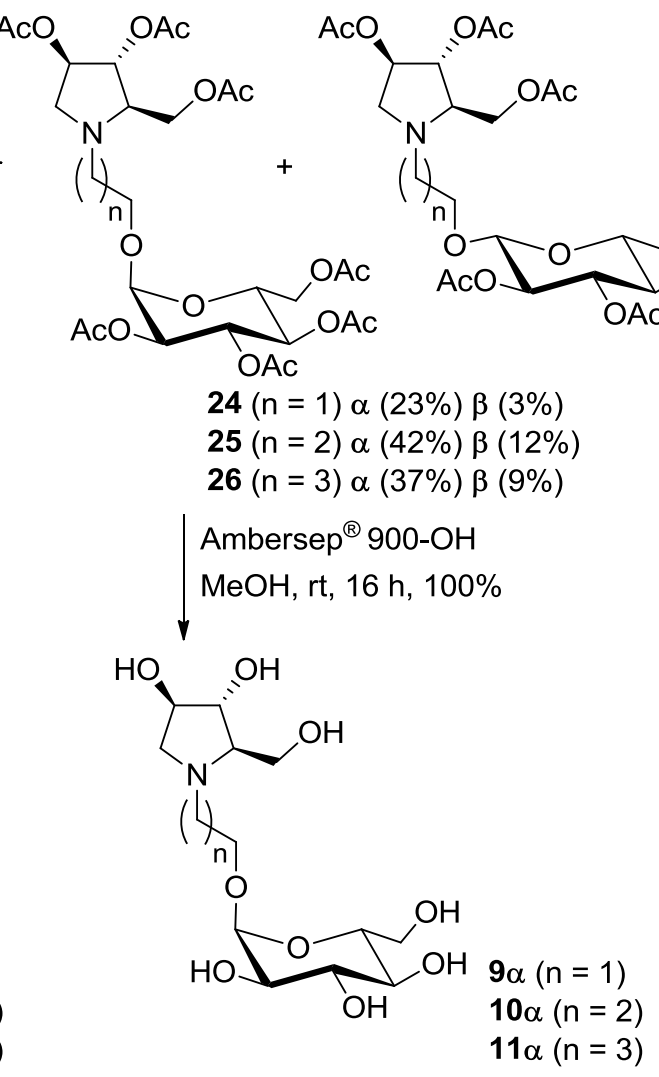

Scheme 3. Synthesis of pseudodisaccharides $\mathbf{9 - 1 1} \alpha$, composed by glucose $1-\alpha$-linked to the pyrrolidine iminosugar DAB-1.

Due to the impossibility to separate the $\alpha$ and $\beta$ isomers both with benzylated and free hydroxyl groups, we decided to directly react the crude obtained by catalytic hydrogenation of $\mathbf{9 - 1 1} \alpha, \beta$ with an excess of acetic anhydride in pyridine affording the corresponding peracetylated compounds 24-26 as $\alpha, \beta$ mixtures. As expected, in all cases, the two isomers were separated by flash column chromatography, affording pure compounds $24 \alpha, 25 \alpha$ and $26 \alpha$ in $23 \%, 42 \%$ and $37 \%$ yields over two steps and $\beta$-isomers $(24 \beta, 25 \beta$ and $26 \beta$ in $3 \%, 12 \%$ and $9 \%$ yield) impure of some traces of the corresponding $\alpha$-isomers (Scheme 3 ). The $\alpha$-isomers $24-26 \alpha$ were subsequently treated with strongly basic Ambersep $900 \mathrm{OH}$ resin in $\mathrm{MeOH}$, leading to pure polyhydroxylated $\alpha$-pseudodisaccharides $\mathbf{9 - 1 1} \alpha$ in quantitative yield. 
Synthesized compounds $8, \mathbf{9} \alpha, \mathbf{1 0} \alpha, \mathbf{1 1} \alpha$ and the $\alpha / \beta$ mixture of compounds $\mathbf{9 , 1 0}$ and 11 were tested for their inhibitory activity against insect (C. riparius) and porcine kidney trehalases and the results are shown in Table 1, together with the previously published data on compounds 4, 6 and 7 [7].

Table 1. Biological activity $\left(\mathrm{IC}_{50}\right)$ towards $\mathrm{C}$. riparius and porcine trehalases.

\begin{tabular}{ccccc}
\hline Entry & Compound & $\begin{array}{c}\text { C. riparius } \\
\text { Trehalase }\end{array}$ & Porcine Trehalase & Selectivity $^{2}$ \\
\hline entry 1 & $\mathbf{4}$ & $44 \pm 1 \mathrm{nM}^{1}$ & $479 \pm 45 \mathrm{nM}^{1}$ & 10 \\
entry 2 & $\mathbf{6}$ & $177 \pm 18 \mathrm{nM}^{1}$ & $>1 \mathrm{mM}^{1}$ & $>5649$ \\
entry 3 & 7 & $175 \pm 12 \mathrm{nM}^{1}$ & $>1 \mathrm{mM}^{1}$ & $>5714$ \\
entry 4 & $\mathbf{8}$ & $29.49 \pm 7.26 \mu \mathrm{M}$ & $190.60 \pm 34.15 \mu \mathrm{M}$ & 6 \\
entry 5 & $\mathbf{9} \alpha, \beta$ & $2.30 \pm 0.13 \mu \mathrm{M}$ & $7.67 \pm 3.91 \mu \mathrm{M}$ & 3 \\
entry 6 & $\mathbf{9} \alpha$ & $9.36 \pm 1.49 \mu \mathrm{M}$ & $27.64 \pm 5.35 \mu \mathrm{M}$ & 3 \\
entry 7 & $\mathbf{9} \beta$ & $0.784 \pm 0.059 \mu \mathrm{M}$ & $5.84 \pm 0.26 \mu \mathrm{M}$ & 7 \\
entry 8 & $\mathbf{1 0} \alpha, \beta$ & $>1000 \mu \mathrm{M}$ & n.d. & - \\
entry 9 & $\mathbf{1 0} \alpha$ & $>1000 \mu \mathrm{M}$ & n.d. & - \\
entry 10 & $\mathbf{1 1} \alpha, \beta$ & $>1000 \mu \mathrm{M}$ & n.d. & - \\
entry 11 & $\mathbf{1 1} \alpha$ & $>1000 \mu \mathrm{M}$ & n.d. & - \\
\hline
\end{tabular}

${ }^{1}$ Value taken from Ref. [7]. ${ }^{2}$ Selectivity is the ratio between $\mathrm{IC}_{50}$ value against porcine trehalase and the $\mathrm{IC}_{50}$ value against $C$. riparius trehalase. ${ }^{3}$ n.d. $=$ not determined.

As already mentioned in the introduction, compounds 6 and 7 , bearing the opposite configuration at C-6 with respect to the pyrrolizidine portion of compound 4, showed a remarkable selectivity (higher than 5000) towards the insect trehalase with respect to the porcine enzyme. However, they were less active (one order of magnitude) than the pseudodisaccharide mimic 4 [7]. For this reason, we planned the synthesis of compound 8 , possessing both a pseudodisaccharide structure and the same configuration at the C-6 carbon atom of compounds 6 and 7. The $\mathrm{IC}_{50}$ value, measured towards insect trehalase, appeared quite disappointing, since compound 8 was active only in the $\mu \mathrm{M}$ range. However, quite a good selectivity was still observed with respect to porcine trehalase (entry 4, Table 1). These results can be rationalized assuming that the active catalytic site of the $C$. riparius trehalase accommodates the pyrrolizidine portion of the compound, as it happens with recombinant Tre37A trehalase, $[5,6]$ : in this case it appears evident that a pyrrolidizine with such configuration at C-6 (such as 8) is not able to place the glucosyl moiety in a part of the enzyme cavity with favorable interactions.

Derivatives 9-11 were designed in order to simplify the overall synthesis of the inhibitors and the data, shown in Table 1, clearly demonstrate that only compounds $\mathbf{9}$ are able to maintain inhibitory properties towards C. riparius trehalase, while compounds with a longer linker chain (e.g., $\mathbf{1 0}$ and 11) loose completely their inhibitory properties (entries 8-11). Collected data suggest that only the two-carbon chain linker of compounds $\mathbf{9}$ is able to mimic the pyrrolizidine moiety of compound $\mathbf{8}$ (see also Figure 3), while its higher flexibility probably allows a better placement of the inhibitor within the active cavity. This is a very good result, which demonstrates the crucial role played by the linker chain's length joining the iminosugar and the glucosyl moiety. Considering that compounds 9 are more active than the pyrrolizidine-based pseudodisaccharide 8 , the advantage of using flexible pyrrolidine-based inhibitors was therefore demonstrated.

Interestingly, the $9 \alpha, \beta$ mixture was more active than compound $9 \alpha$ alone (entry 5 vs. entry 6 , Table 1). Thus, we reasoned that the pure $\beta$-anomer might be even more active. In order to obtain a substantial amount of the $\beta$-isomer $9 \beta$, we decided to change the protecting groups on the glycosyl donor by employing the $O$-acetyl protected 1- $\alpha$-trichloroacetimidate 29 (Scheme 4), which would in principle favor the formation of $\beta$-pseudodisaccharide 30 in the following glycosylation reaction through the orthoester procedure (1,2-trans glycosylation) $[15,16]$.

Selective deprotection of $\mathbf{2 7}$ with ethylendiamine in acetic acid following the procedure reported by Sun et al. [17] afforded in $86 \%$ yield compound 28 , that was treated with trichloroacetonitrile to obtain 29 in $85 \%$ yield. Glycosylation reaction with 15 at $0{ }^{\circ} \mathrm{C}$ in dry $\mathrm{CH}_{2} \mathrm{Cl}_{2}$ in the presence of TMSOTF (1.5 equiv) afforded 39\% yield of the $\beta$-glucoside $\mathbf{3 0}$ as the major compound, although impure of 
a partially deacetylated isomer (See Supplementary Materials). The ${ }^{1} \mathrm{H}-\mathrm{NMR}$ spectrum of compound 30 showed a $d d$ at 5.05 ppm for H-2' signal (appearing as a pseudo triplet), with coupling constants of 9.6 and $9.8 \mathrm{~Hz}$. This indicates an axial-axial relationship with both $\mathrm{H}-\mathrm{1}^{\prime}$ and $\mathrm{H}-3^{\prime}$, and therefore confirms the $\beta$-configuration of the glucose moiety.

Deprotection of the benzyl groups by catalytic hydrogenation and of the acetyl groups by treatment with Ambersep 900-OH, allowed to isolate pure disaccharide $9 \beta$ in $43 \%$ yield over 2 steps (Scheme 4). To our delight and accordingly to our expectation, compound $9 \beta$ was 12 -fold more active than its $\alpha$-anomer towards $C$. riparius trehalase and was the most potent insect trehalase inhibitor of the pseudodisaccharide pyrrolidine series, with an $\mathrm{IC}_{50}$ in the low micromolar range $\left(\mathrm{IC}_{50}=0.784 \mu \mathrm{M}\right.$, entry 7, Table 1). Moreover, $9 \beta$ showed also a good 7-fold selectivity towards the insect trehalase vs. the porcine enzyme.
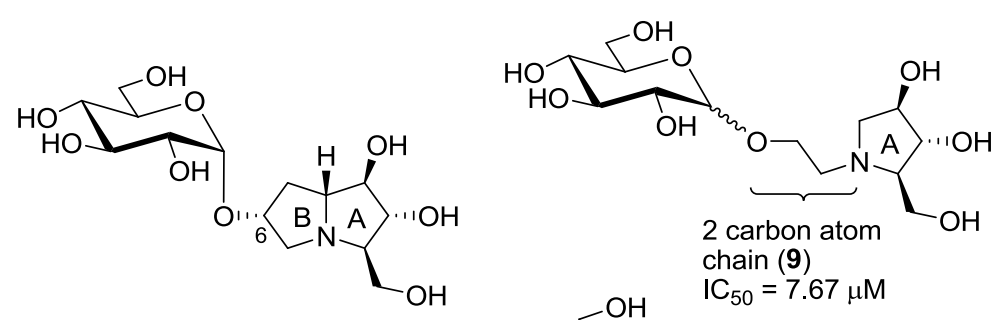

7-deoxycasuarine glucoside (4) $\mathrm{IC}_{50}=44 \mathrm{nM}$

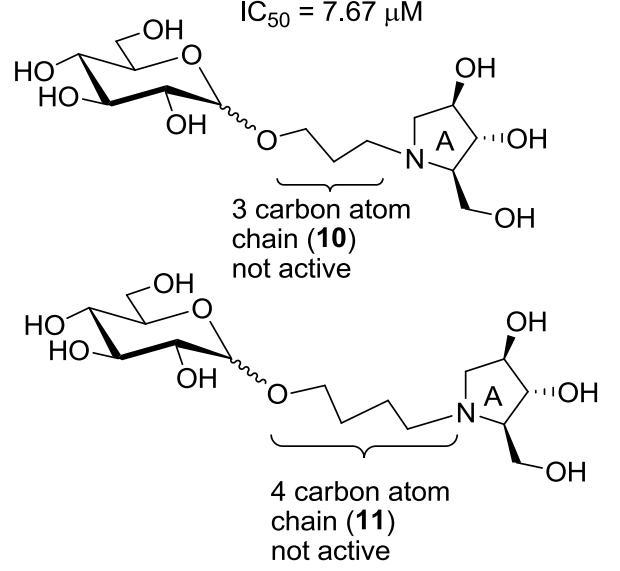

Figure 3. Compounds 4, 8, 9, 10, 11 and their $\mathrm{IC}_{50}$ values towards $C$. riparius trehalase.
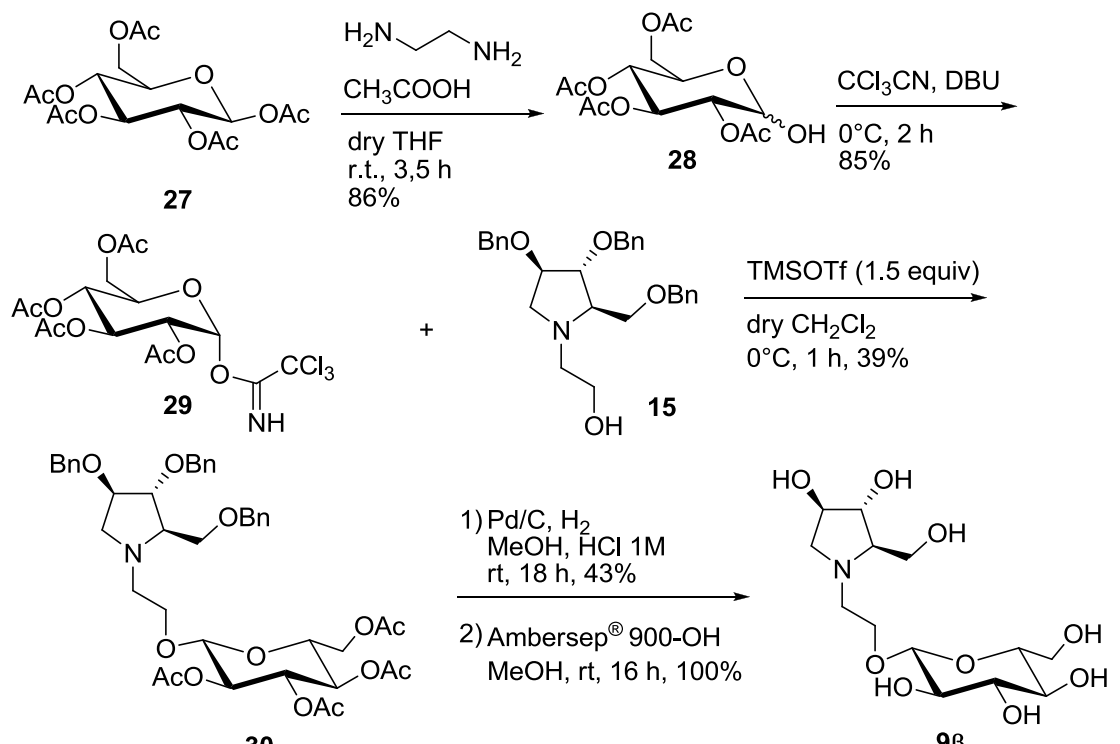

30

$9 \beta$

Scheme 4. Synthesis of the pseudodisaccharide $9 \beta$. 
To evaluate the inhibitory pattern we performed kinetics measurements by varying the trehalose concentration, at two different concentrations of compounds $9 \alpha$ and $9 \beta$. Results showed a pure competitive inhibitory pattern. To determine the inhibition type, data were plotted according to the Lineweaver-Burk plot, replots were built and an inhibition constant $\left(\mathrm{K}_{\mathrm{i}}\right)$ was calculated equal to $2.56 \pm 0.42 \mu \mathrm{M}$ for $9 \alpha$ and $0.40 \pm 0.022 \mu \mathrm{M}$ for $9 \beta$ (see supplementary materials).

\section{Materials and Methods}

\subsection{General Experimental Procedures}

All the starting reactants, solvents, and catalysts were commercially available unless otherwise stated. All reactions were carried out under magnetic stirring and monitored by TLC on $0.25 \mathrm{~mm}$ silica gel plates (Merck F254). Column chromatographies were carried out on Silica Gel 60 (32-63 $\mu \mathrm{m})$ or on silica gel (230-400 mesh, Merck, Kenilworth, NJ, USA). Yields refer to spectroscopically and analytically pure compounds unless otherwise stated. ${ }^{1} \mathrm{H}-\mathrm{NMR}$ spectra were recorded on a Varian Mercury-400 or on a Varian INOVA 400 instruments (Agilent Technologies, Santa Clara, CA, USA) at $25{ }^{\circ} \mathrm{C}$. ${ }^{13} \mathrm{C}-\mathrm{NMR}$ spectra were recorded on a Varian Gemini-200 spectrometer (Agilent Technologies, Santa Clara, CA, USA). Chemical shifts are reported relative to TMS $\left({ }^{1} \mathrm{H}: \delta=0.00 \mathrm{ppm}\right)$ and $\mathrm{CDCl} 3$ $\left({ }^{13} \mathrm{C}: \delta=77.0 \mathrm{ppm}\right)$. Integrals are in accordance with assignments, coupling constants are given in Hz. For detailed peak assignments 2D spectra were measured (COSY, HSQC, NOESY, and NOE as necessary). IR spectra were recorded with a BX FTIR Perkin-Elmer system spectrophotometer (Perkin-Elmer, Waltham, MA, USA). ESIMS spectra were recorded with a Thermo Scientific ${ }^{\mathrm{TM}}$ LCQ fleet ion trap mass spectrometer (Thermo Fisher Scientific, Waltham, MA, USA). Elemental analyses were performed with a Perkin-Elmer 2400 analyzer (Perkin-Elmer, Waltham, MA, USA). Optical rotation measurements were performed on a JASCO DIP-370 polarimeter (JASCO, Easton, MD, USA).

\subsection{Synthesis and Purification of 7-Deoxyuniflorine A Glucoside (8)}

\subsubsection{Synthesis of Compounds $\mathbf{1 8}$}

To a solution of 2,3,4,6-tetra-O-benzyl-D-glucopyranose (300 mg, $0.55 \mathrm{mmol}$ ) in $7 \mathrm{~mL}$ of dry $\mathrm{CH}_{3} \mathrm{CN}$, trichloroacetonitrile $(568 \mu \mathrm{L}, 7.15 \mathrm{mmol})$ and $\mathrm{K}_{2} \mathrm{CO}_{3}(380 \mathrm{mg}, 2.75 \mathrm{mmol})$ were added under nitrogen atmosphere and the mixture was stirred at room temperature for $4 \mathrm{~h}$. When a TLC analysis $\left(\mathrm{CH}_{2} \mathrm{Cl}_{2} / \mathrm{MeOH} 30: 1\right)$ showed the disappearance of the starting material $\left(R_{\mathrm{f}}=0.06\right)$ and formation of a new product $\left(R_{\mathrm{f}}=0.33\right)$, the mixture was filtered through Celite ${ }^{\circledR}$ and the solvent was removed under reduced pressure affording a crude oil. The crude, obtained in a quantitative yield, was used directly for the next step without further purification.

\subsubsection{Synthesis of Compounds 19}

A solution of alcohol 13 [7] (100 mg, $0.21 \mathrm{mmol})$ and glucopyranosyl tricholoroacetimidate $18(230 \mathrm{mg}, 0.35 \mathrm{mmol})$ in $4.2 \mathrm{~mL}$ of dry $\mathrm{Et}_{2} \mathrm{O}$ was stirred for $10 \mathrm{~min}$ at room temperature under nitrogen atmosphere in the presence of $3 \AA$ molecular sieves. After cooling to $-20^{\circ} \mathrm{C}$ and addition of trimethylsilyl trifluoromethanesulfonate $(20 \mu \mathrm{L}, 0.11 \mathrm{mmol})$, stirring was continued for $1 \mathrm{~h}$; during this period the temperature was raised to room temperature. A TLC analysis (PE/EtOAc 1:1) showed the disappearance of the starting material $\left(R_{\mathrm{f}}=0.22\right)$ and formation of two new products $\left(R_{\mathrm{f}}=0.72\right.$ and 0.66). Then $2 \mathrm{~mL}$ of a saturated $\mathrm{Na}_{2} \mathrm{CO}_{3}$ solution were added, the mixture was extracted with $\mathrm{Et}_{2} \mathrm{O}(2 \times 15 \mathrm{~mL})$ and the organic layers were dried on $\mathrm{Na}_{2} \mathrm{SO}_{4}$. Filtration on cotton and evaporation under reduced pressure afforded the crude 19 that was purified by flash column chromatography on silica gel (PE/EtOAc from 3:1 to 1:1) to afford pure 19 $\beta\left(R_{\mathrm{f}}=0.25 \mathrm{PE} / \mathrm{EtOAc} 3: 1,9 \mathrm{mg}, 0.009 \mathrm{mmol}\right.$, $4 \%$ yield $)$ and $19 \alpha\left(R_{\mathrm{f}}=0.18 \mathrm{PE} /\right.$ EtOAc 3:1, $179 \mathrm{mg}, 0.18 \mathrm{mmol}, 86 \%$ yield $)$ as yellow oils.

(1R,2R,3R,6S,7aR)-1,2-bis(benzyloxy)-3-[(benzyloxy)methyl]-5-oxohexahydro-1H-pyrrolizin-6-yl 2,3,4,6-tetraO-benzyl- $\alpha$-hexopyranoside. $19 \alpha:[\alpha]_{\mathrm{D}}^{18}=+18.3\left(c=0.06, \mathrm{CHCl}_{3}\right) .{ }^{1} \mathrm{H}-\mathrm{NMR}\left(400 \mathrm{MHz}, \mathrm{CDCl}_{3}\right)$ : 
$\delta=7.38-7.11(\mathrm{~m}, 35 \mathrm{H}, \mathrm{H}-\mathrm{Ar}), 4.92-4.40(\mathrm{~m}, 14 \mathrm{H}, \mathrm{H}-\mathrm{Bn}), 4.83-4.80(\mathrm{~m}, 1 \mathrm{H}, \mathrm{H}-1), 4.33(\mathrm{dd}, J=4.7$, 4.1, 1H, H-1'), 4.25-4.23 (m, 1 H, H-6'), 4.08-4.02 (m, 3 H, H-7a', H-5, H-3'), 3.95 (t, J = 9.4 Hz, 1H, H-3), 3.77 (dd, $\left.J=10.8,2.6 \mathrm{~Hz}, 1 \mathrm{H}, \mathrm{Ha}-8^{\prime}\right), 3.72$ (t, $\left.J=9.7 \mathrm{~Hz}, 1 \mathrm{H}, \mathrm{H}-4\right), 3.68-3.65$ (m, $\left.1 \mathrm{H}, \mathrm{Hb}-8^{\prime}\right)$, 3.63-3.56 (m, 4 H, H-2, H-2' , H-6), 2.23-2.18 (m, 1 H, Ha-7'), 1.95 (dt, $J=13.8,6.7$ Hz, 1 H, Hb-7') ppm. ${ }^{13} \mathrm{C}-\mathrm{NMR}\left(50 \mathrm{MHz}, \mathrm{CDCl}_{3}\right): 171.3$ (s, C=O), 139.1-137.6 (s, $\left.7 \mathrm{C}, \mathrm{C}-\mathrm{Ar}\right), 128.6-127.6$ (d, $35 \mathrm{C}$, C-Ar), $96.7(\mathrm{~d}, \mathrm{C}-1), 88.8\left(\mathrm{~d}, \mathrm{C}-2^{\prime}\right), 87.2\left(\mathrm{~d}, \mathrm{C}-1^{\prime}\right), 82.0(\mathrm{~d}, \mathrm{C}-3), 80.2(\mathrm{~d}, \mathrm{C}-2), 77.8\left(\mathrm{~d}, \mathrm{C}-6^{\prime}\right), 77.2(\mathrm{~d}, \mathrm{C}-4)$, 76.5-72.4 (t, 7 C, C-Bn), 70.5 (d, C-3'), 69.2 (t, C-6), 68.3 (t, C-8), 62.0 (d, C-7a'), 58.9 (d, C-5), 33.3 (t, C-7') ppm. MS (ESI): $m / z(\%)=1018.75(100)[\mathrm{M}+\mathrm{Na}]^{+}$. IR $\left(\mathrm{CDCl}_{3}\right): 3088,3066,3032,2915,2867,1700$, $1602,1496,1453,1363,1098,1071,1028 \mathrm{~cm}^{-1}$. Elemental analysis (\%) for $\mathrm{C}_{63} \mathrm{H}_{65} \mathrm{NO}_{10}$ (996.19): calcd. C 75.96, H 6.58, N 1.41; found C 75.92, H 6.55, N 1.43.

$(1 R, 2 R, 3 R, 6 S, 7 a R)-1,2-$ bis(benzyloxy)-3-[(benzyloxy)methyl]-5-oxohexahydro-1H-pyrrolizin-6-yl 2,3,4,6-tetraO-benzyl- $\beta$-hexopyranoside. 19 $\beta$ : $[\alpha]_{\mathrm{D}}^{19}=-40.0\left(c=0.04, \mathrm{CHCl}_{3}\right) .{ }^{1} \mathrm{H}-\mathrm{NMR}\left(400 \mathrm{MHz}, \mathrm{CDCl}_{3}\right)$ : $\delta=7.36-7.12(\mathrm{~m}, 35 \mathrm{H}, \mathrm{H}-\mathrm{Ar}), 4.91-4.49$ (m, $14 \mathrm{H}, \mathrm{H}-\mathrm{Bn}), 4.82-4.79(\mathrm{~m}, 1 \mathrm{HH}-1), 4.46-4.44(\mathrm{~m}, 1 \mathrm{H}$, $\left.\mathrm{H}-6^{\prime}\right), 4.32\left(\mathrm{dd}, J=4.7,3.8,1 \mathrm{H}, \mathrm{H}-1^{\prime}\right), 4.10-4.04\left(\mathrm{~m}, 2 \mathrm{H}, \mathrm{H}-7 \mathrm{a}^{\prime}\right.$ and H-5), 3.70-3.59 (m, $5 \mathrm{H}, \mathrm{H}-2^{\prime}, \mathrm{H}-3$, H-4 and H-8), 3.50-3.39 (m, 4 H, H-3', H-2 and H-6), 2.46-2.41 (m, 1 H, Ha-7'), 2,04 (dt, J = 14.7, 7.0 Hz, $\left.1 \mathrm{H}, \mathrm{Hb}-7^{\prime}\right)$ ppm. ${ }^{13} \mathrm{C}-\mathrm{NMR}\left(100 \mathrm{MHz}, \mathrm{CDCl}_{3}\right): 171.5$ (s, C=O), 138.6-137.5 (s, $\left.7 \mathrm{C}, \mathrm{C}-\mathrm{Ar}\right), 128.5-127.5$ (d, 35 C, C-Ar), $102.2(\mathrm{~d}, \mathrm{C}-1), 88.8\left(\mathrm{~d}, \mathrm{C}-2^{\prime}\right), 87.3\left(\mathrm{~d}, \mathrm{C}-1^{\prime}\right), 84.5(\mathrm{~d}, \mathrm{C}-4), 81.8(\mathrm{~d}, \mathrm{C}-2), 78.6\left(\mathrm{~d}, \mathrm{C}-6^{\prime}\right), 77.3(\mathrm{~d}$, C-3), 75.7-72.2 (t, 7 C, C-Bn), $74.3\left(\mathrm{~d}, \mathrm{C}-3^{\prime}\right), 69.0(\mathrm{t}, \mathrm{C}-6), 68.7(\mathrm{t}, \mathrm{C}-8), 62.4\left(\mathrm{~d}, \mathrm{C}-7 \mathrm{a}^{\prime}\right), 58.5(\mathrm{~d}, \mathrm{C}-5), 34.8(\mathrm{t}$, C-7') ppm. MS (ESI): $m / z(\%)=1018.58(100)[\mathrm{M}+\mathrm{Na}]^{+}$. IR $\left(\mathrm{CDCl}_{3}\right): 3089,3066,3032,2905,2868,1697$, $1602,1496,1454,1362,1095,1069,1028 \mathrm{~cm}^{-1}$. Elemental analysis (\%) for $\mathrm{C}_{63} \mathrm{H}_{65} \mathrm{NO}_{10}$ (996.19): calcd. C 75.96, H 6.58, N 1.41; found C 75.94, H 6.57, N 1.41.

\subsubsection{Synthesis of Compound 20}

A solution of $19 \alpha(118 \mathrm{mg}, 0.12 \mathrm{mmol})$ in $1.2 \mathrm{~mL}$ of dry THF was stirred under nitrogen atmosphere at $0{ }^{\circ} \mathrm{C}$ and $\mathrm{LiAlH}_{4}(1 \mathrm{M}$ solution in THF, $0.56 \mathrm{~mL}, 0.56 \mathrm{mmol})$ was added drop wise. The mixture was then heated at reflux temperature for $1.5 \mathrm{~h}$, until a TLC analysis (PE/EtOAc 2:1) showed the disappearance of the starting material $\left(R_{\mathrm{f}}=0.57\right)$ and the formation of a new product $\left(R_{\mathrm{f}}=0.75\right)$. The reaction was then quenched at $0{ }^{\circ} \mathrm{C}$ with a saturated aqueous solution of $\mathrm{Na}_{2} \mathrm{SO}_{4}(5 \mathrm{~mL})$. After extraction with EtOAc $(3 \times 15 \mathrm{~mL})$, the organic layers were dried on $\mathrm{Na}_{2} \mathrm{SO}_{4}$ and concentrated at reduced pressure affording the crude that was purified by flash column chromatography on silica gel (PE/EtOAc 4:1) to afford 20 pure $\left(R_{\mathrm{f}}=0.25,108 \mathrm{mg}, 0.11 \mathrm{mmol}, 90 \%\right.$ yield) as a waxy white solid.

(1R,2R,3R,6S,7aR)-1,2-bis(benzyloxy)-3-[(benzyloxy)methyl]hexahydro-1H-pyrrolizin-6-yl 2,3,4,6-tetra-Obenzyl- $\alpha$-hexopyranoside 20: $[\alpha]]_{\mathrm{D}}^{22}=+50.6\left(c=0.17, \mathrm{CHCl}_{3}\right) .{ }^{1} \mathrm{H}-\mathrm{NMR}\left(400 \mathrm{MHz}, \mathrm{CDCl}_{3}\right): \delta=7.36-7.12(\mathrm{~m}$, $35 \mathrm{H}, \mathrm{H}-\mathrm{Ar}), 4.98-4.39$ (m, $14 \mathrm{H}, \mathrm{H}-\mathrm{Bn}), 4.84-4.80$ (m, 1H, H-1), 4.36-4.30 (m, 1H, H-6), 4.03 (t, J = 5.3 Hz, $\left.1 \mathrm{H}, \mathrm{H}-2^{\prime}\right), 3.96-3.90$ (m, 1 H, H-1'), 3.84-3.63 (m, 5 H, H-4, H-5, H-3, H-7a', Ha-6), 3.59-3.54 (m, $3 \mathrm{H}$, 2-H, Hb-6, Ha-8), 3.49 (dd, J = 9.4, 5.9 Hz, 1 H, Hb-8), 3.16 (dd, J = 11.7, 3.5 Hz, 1 H, Ha- 5'), 3.01 (dd, $\left.J=12.3,5.3 \mathrm{~Hz}, 1 \mathrm{H}, \mathrm{Hb}-5^{\prime}\right), 2.94\left(\mathrm{q}, J=5.9 \mathrm{~Hz}, 1 \mathrm{H}, \mathrm{H}-3^{\prime}\right), 2.09-2.05\left(\mathrm{~m}, 1 \mathrm{H}, \mathrm{Ha}-7^{\prime}\right), 1.82(\mathrm{ddd}, J=13.5$, 7.6, $\left.5.9 \mathrm{~Hz}, 1 \mathrm{H}, \mathrm{Hb}-7^{\prime}\right)$ ppm. ${ }^{13} \mathrm{C}-\mathrm{NMR}\left(50 \mathrm{MHz}, \mathrm{CDCl}_{3}\right)$ : 139.1-138.1 (s, $\left.7 \mathrm{C}, \mathrm{C}-\mathrm{Ar}\right), 128.4-127.0$ (d, 35 C, C-Ar), $96.5(\mathrm{~d}, \mathrm{C}-1), 88.5(\mathrm{~d}, \mathrm{C}-3), 86.1\left(\mathrm{~d}, \mathrm{C}-2^{\prime}\right), 82.0\left(\mathrm{~d}, \mathrm{C}-1^{\prime}\right), 80.3(\mathrm{~d}, \mathrm{C}-2), 78.4\left(\mathrm{t}, \mathrm{C}-6^{\prime}\right), 77.9(\mathrm{~d}$, C-4), 75.7-71.8 (t, 8 C, C-Bn and C-8), $70.6(\mathrm{~d}, \mathrm{C}-5), 69.4\left(\mathrm{~d}, \mathrm{C}-3^{\prime}\right), 68.6(\mathrm{t}, \mathrm{C}-6), 66.4\left(\mathrm{~d}, \mathrm{C}-7 \mathrm{a}^{\prime}\right), 60.6(\mathrm{t}$, C-5'), $37.0\left(\mathrm{t}, \mathrm{C}-7^{\prime}\right)$ ppm. MS (ESI): $m / z(\%)=982.50(100)[\mathrm{M}+\mathrm{H}]^{+}$. IR $\left(\mathrm{CDCl}_{3}\right): 3089,3067,3032,2915$, $2867,1605,1496,1454,1363,1144,1070,1028 \mathrm{~cm}^{-1}$. Elemental analysis (\%) for $\mathrm{C}_{63} \mathrm{H}_{67} \mathrm{NO}_{9}$ (982.21): calcd. C 77.04, H 6.88, N 1.43; found C 77.01, H 6.87, N 1.41.

\subsubsection{Synthesis of Compound 8}

To a solution of compound 20 (153 mg, $0.16 \mathrm{mmol})$ in $15 \mathrm{~mL}$ of methanol, 10\% Pd/C (77 mg, $50 \%$ weight) and two drops of $37 \% \mathrm{HCl}$ were added under nitrogen atmosphere, then the mixture was stirred under hydrogen atmosphere at room temp for three days. At completion of the reaction, the mixture was filtered through Celite ${ }^{\circledR}$ and the solvent was removed under reduced pressure 
affording a crude oil. Free amine 8 ( $53 \mathrm{mg}, 0.15 \mathrm{mmol}, 93 \%$ yield) was obtained by purification with ion exchange resin Dowex 50WX8-200, eluting successively with $\mathrm{MeOH}, \mathrm{H}_{2} \mathrm{O}$ and $6 \% \mathrm{NH}_{4} \mathrm{OH}$.

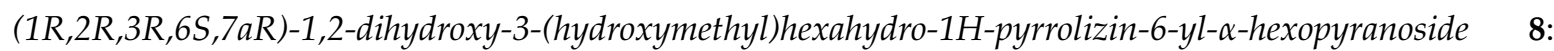
$[\alpha]_{\mathrm{D}}^{22}=+102.3\left(c=0.39, \mathrm{H}_{2} \mathrm{O}\right) .{ }^{1} \mathrm{H}-\mathrm{NMR}\left(400 \mathrm{MHz}, \mathrm{H}_{2} \mathrm{O}\right): \delta=4.78(\mathrm{~d}, J=3.9 \mathrm{~Hz}, 1 \mathrm{H}, \mathrm{H}-1), 4.36-4.30(\mathrm{~m}$, 1H, H-6'), 3.68-3.63 (m, 2 H, H-1' , H-2'), 3.63-3.44 (m, 4 H, H-4, H-5, H-6), 3.57 (dd, J = 11.7, 3.9 Hz, 1H, Ha-8), 3.41 (dd, $J=11.7,6.9$ Hz, $1 \mathrm{H}, \mathrm{Hb}-8), 3.30$ (dd, $J=9.8,3.5 \mathrm{~Hz}, 1 \mathrm{H}, \mathrm{H}-2), 3.18-3.12$ (m, $\left.1 \mathrm{H}, \mathrm{H}-7 \mathrm{a}^{\prime}\right), 3.16(\mathrm{t}, J=9.7 \mathrm{~Hz}, 1 \mathrm{H}, \mathrm{H}-3), 3.01-2.98\left(\mathrm{~m}, 1 \mathrm{H}, \mathrm{Ha}-5^{\prime}\right), 2.76(\mathrm{dd}, J=11.7,3.9 \mathrm{~Hz}, 1 \mathrm{H}$, Hb-5'), 2.63-2.56 (m, $\left.1 \mathrm{H}, \mathrm{H}-3^{\prime}\right), 2.13-2.07\left(\mathrm{~m}, 1 \mathrm{H}, \mathrm{Ha}-7^{\prime}\right), 1.71\left(\mathrm{ddd}, J=13.2,8.3,4.9,1 \mathrm{H}, \mathrm{Hb}-7^{\prime}\right)$ ppm. ${ }^{13} \mathrm{C}-\mathrm{NMR}\left(100 \mathrm{MHz}, \mathrm{H}_{2} \mathrm{O}\right): 96.0$ (d, C-1), 80.3 (d, C-1'), 78.0 (d, C-6'), 77.6 (d, C-2'), 72.9 (d, C-4), 71.9 (d, C-5), $71.1(\mathrm{~d}, \mathrm{C}-2), 70.1\left(\mathrm{~d}, \mathrm{C}-3^{\prime}\right), 69.5(\mathrm{~d}, \mathrm{C}-3), 64.9\left(\mathrm{~d}, \mathrm{C}-7 \mathrm{a}^{\prime}\right), 62.5(\mathrm{t}, \mathrm{C}-8), 60.6(\mathrm{t}, \mathrm{C}-6), 60.5\left(\mathrm{t}, \mathrm{C}-5^{\prime}\right)$, $34.2\left(\mathrm{t}, \mathrm{C}-7^{\prime}\right)$ ppm. MS (ESI): $m / z(\%)=374.25(100)[\mathrm{M}+\mathrm{Na}]^{+}$. Elemental analysis (\%) for $\mathrm{C}_{14} \mathrm{H}_{25} \mathrm{NO}_{9}$ (351.15): calcd. C 47.86, H 7.17, N 3.99; found C 47.83, H 7.16, N 3.98.

\subsection{Synthesis and Purification of Pyrrolidine-based Pseudodisacharides $\mathbf{9} \alpha, \mathbf{1 0} \alpha$ and $\mathbf{1 1} \alpha$}

\subsubsection{Synthesis of Compound 15}

To a solution of 14 [13] $(82 \mathrm{mg}, 0.20 \mathrm{mmol})$ in $3 \mathrm{~mL}$ of THF, NEt $3(141 \mu \mathrm{L}, 1.00 \mathrm{mmol})$ and 2-bromo-1-etanol ( $85 \mu \mathrm{L}, 1.22 \mathrm{mmol}$ ) were added. The reaction mixture was stirred at room temperature for 3 days until a TLC analysis $\left(\mathrm{CH}_{2} \mathrm{Cl}_{2}: \mathrm{MeOH} 30: 1\right)$ showed the disappearance of the starting material $\left(R_{f}=0.43\right)$ and the formation of a new product $\left(R_{f}=0.81\right)$. After evaporation under reduced pressure, the crude was purified by FCC (AcOEt:PE 1:1) affording pure $15\left(\mathrm{R}_{\mathrm{f}}=0.30,75 \mathrm{mg}, 0.17 \mathrm{mmol}, 85 \%\right.$ yield) as a yellow oil.

2-\{(2R,3R,4R)-3,4-bis(benzyloxy)-2-[(benzyloxy)methyl]-1-[(2-hydroxy)etyl]-1H-pyrrolidine 15 [12]: $[\alpha]_{\mathrm{D}}^{25}=-20.3\left(c=0.92\right.$ in $\left.\mathrm{CHCl}_{3}\right) ;{ }^{1} \mathrm{H}-\mathrm{NMR}\left(400 \mathrm{MHz}, \mathrm{CDCl}_{3}\right): \delta=7.35-7.26(\mathrm{~m}, 15 \mathrm{H}, \mathrm{H}-\mathrm{Ar})$, 4.55-4.42 (m, 6H, H-Bn), 3.99-3.97 (m, 1H, H-4), 3.89 (dd, J = 3.6, 1.2 Hz, 1H, H-3), 3.64-3.48 (m, 4H, H-7, H-8), 3.25 (d, J = 10.4 Hz, 1H, Ha-5), 3.06 (ddd, J = 12.9, 9.1, 4.7 Hz, 1H, Ha-6), 2.88 (dd, J = 9.6, $5.6 \mathrm{~Hz}, 1 \mathrm{H}, \mathrm{H}-2), 2.67$ (dd, $J=10.4,5.2 \mathrm{~Hz}, 1 \mathrm{H}, \mathrm{Hb}-5), 2.58$ (dt, $J=12.6,3.8 \mathrm{~Hz}, 1 \mathrm{H}, \mathrm{Hb}-6)$.

\subsubsection{Synthesis of Compound $\mathbf{1 6}$}

To a solution of 14 [13] (132 mg, $0.33 \mathrm{mmol})$ in $6 \mathrm{~mL}$ of THF, NEt $3(230 \mu \mathrm{L}, 1.65 \mathrm{mmol})$ and 3-bromo-1-propanol $(179 \mu \mathrm{L}, 1.98 \mathrm{mmol})$ were added. The reaction mixture was stirred at room temperature for 3 days until a TLC analysis $\left(\mathrm{CH}_{2} \mathrm{Cl}_{2}\right.$ :MeOH 10:1) showed the disappearance of the starting material $\left(R_{f}=0.51\right)$ and the formation of a new product $\left(R_{f}=0.85\right)$. After evaporation under reduced pressure, the crude was purified by FCC (AcOEt:PE 1:1) affording pure $\mathbf{1 6}\left(\mathrm{R}_{\mathrm{f}}=0.30,143 \mathrm{mg}\right.$, $0.31 \mathrm{mmol}, 94 \%$ yield) as a yellow oil.

(2R,3R,4R)-3,4-bis(benzyloxy)-2-[(benzyloxy)methyl]-1-[(3-hydroxy)propyl]-1H-pyrrolidine 16: $[\alpha]_{\mathrm{D}}^{24}=-39.1$ $\left(c=0.47\right.$ in $\left.\mathrm{CHCl}_{3}\right) ;{ }^{1} \mathrm{H}-\mathrm{NMR}\left(400 \mathrm{MHz}, \mathrm{CDCl}_{3}\right): \delta=7.34-7.24(\mathrm{~m}, 15 \mathrm{H}, \mathrm{H}-\mathrm{Ar}), 4.56-4.48(\mathrm{~m}, 6 \mathrm{H}$, H-Bn), 3.93 (d, $J=4.8 \mathrm{~Hz}, 1 \mathrm{H}, \mathrm{H}-4), 3.82-3.77$ (m, 3H, H-9 and H-3), 3.63 (dd, $J=9.7,5.8 \mathrm{~Hz}, 1 \mathrm{H}, \mathrm{Ha}-6)$, $3.55(\mathrm{dd}, J=9.8,6.4 \mathrm{~Hz}, 1 \mathrm{H}, \mathrm{Hb}-6), 3.45(\mathrm{~d}, J=10.8 \mathrm{~Hz}, 1 \mathrm{H}, \mathrm{Ha}-5), 3.14(\mathrm{td}, J=11.7,3.9 \mathrm{~Hz}, 1 \mathrm{H}, \mathrm{Ha}-7)$, $2.73(\mathrm{q}, J=5.2 \mathrm{~Hz}, 1 \mathrm{H}, \mathrm{H}-2), 2.64(\mathrm{dt}, J=12.2,3.9 \mathrm{~Hz}, 1 \mathrm{H}, \mathrm{Hb}-7), 2.50(\mathrm{dd}, J=10.8,4.7 \mathrm{~Hz}, 1 \mathrm{H}, \mathrm{Hb}-5)$, 1.94-1.82 (m, 1H, Ha-8), 1.56-1.49 (m, 1H, Hb-8); ${ }^{13} \mathrm{C}-\mathrm{NMR}\left(50 \mathrm{MHz}, \mathrm{CDCl}_{3}\right): \delta=138.3,138.2,138.1$ (s, 3C, C-Ar), 128.3-127.5 (d, 15C, C-Ar), 85.3 (d, C-3), 81.4 (d, C-4), 73.3, 71.4, 71.2, 70.9 (t, 4C, C-Bn and C-6), 69.7 (d, C-2), 64.0 (t, C-9), $56.7(\mathrm{t}, \mathrm{C}-5), 55.2(\mathrm{t}, \mathrm{C}-7), 29.3(\mathrm{t}, \mathrm{C}-8)$; IR $\left(\mathrm{CDCl}_{3}\right): v=3312,3066$, 3032, 2923, 2861, 1496, 1453, 1366, 1333, 1282, 1208, 1100, 1071, $1028 \mathrm{~cm}^{-1}$; MS (ESI): $\mathrm{m} / z$ calcd (\%) for $\mathrm{C}_{29} \mathrm{H}_{35} \mathrm{NO}_{4}+\mathrm{Na}^{+} 484.26[\mathrm{M}+\mathrm{Na}]^{+}$; found: 484.33 ; elemental analysis calcd (\%) for $\mathrm{C}_{29} \mathrm{H}_{35} \mathrm{NO}_{4}$ (461.59): C 75.46, H 7.64, N 3.03; found: 75.43, H 7.62, N 3.02. 


\subsubsection{Synthesis of Compound $\mathbf{1 7}$}

To a solution of 14 [13] (100 mg, $0.25 \mathrm{mmol})$ in $1 \mathrm{~mL}$ of THF, NEt $3(174 \mu \mathrm{L}, 1.25 \mathrm{mmol})$ and 4-bromo-1-butanol $(158 \mu \mathrm{L}, 1.5 \mathrm{mmol})$ were added. The reaction mixture was stirred at room temperature for 3 days until a TLC analysis $\left(\mathrm{CH}_{2} \mathrm{Cl}_{2}: \mathrm{MeOH} 10: 1\right)$ showed the disappearance of the starting material $\left(R_{\mathrm{f}}=0.51\right)$ and the formation of a new product $\left(R_{\mathrm{f}}=0.65\right)$. After evaporation under reduced pressure, the crude was purified by FCC (AcOEt:PE 2:1) affording pure $17\left(R_{\mathrm{f}}=0.35\right.$, $105 \mathrm{mg}, 0.22 \mathrm{mmol}, 88 \%$ yield) as a yellow oil.

$(2 R, 3 R, 4 R)-3,4-b i s\left(\right.$ benzyloxy)-2-[(benzyloxy)methyl]-1-[(4-hydroxy)butyl]-1H-pyrrolidine 17: $[\alpha]_{\mathrm{D}}^{25}=-26.2$ $\left(c=0.60\right.$ in $\left.\mathrm{CHCl}_{3}\right) ;{ }^{1} \mathrm{H}-\mathrm{NMR}\left(400 \mathrm{MHz}, \mathrm{CDCl}_{3}\right): \delta=7.34-7.24(\mathrm{~m}, 15 \mathrm{H}, \mathrm{H}-\mathrm{Ar}), 4.56-4.39(\mathrm{~m}, 6 \mathrm{H}$, H-Bn), $3.94(\mathrm{~d}, J=4.8 \mathrm{~Hz}, 1 \mathrm{H}, \mathrm{H}-4), 3.86$ (d, J = 3.9 Hz, 1H, H-3), 3.67-3.63 (m, 2H, Ha-10 and Ha-6), 3.57-3.51 (m, 2H, Hb-10 and Hb-6), $3.30(\mathrm{~d}, J=10.8 \mathrm{~Hz}, 1 \mathrm{H}, \mathrm{Ha}-5), 2.94-2.87(\mathrm{~m}, 1 \mathrm{H}, \mathrm{Ha}-7), 2.81-2.77(\mathrm{~m}$, $1 \mathrm{H}, \mathrm{H}-2), 2.54$ (dd, $J=10.7,4.8 \mathrm{~Hz}, 1 \mathrm{H}, \mathrm{Hb}-5), 2.46$ (dt, $J=11.7,4.4 \mathrm{~Hz}, 1 \mathrm{H}, \mathrm{Hb}-7), 1.76-1.66$ (m, 2H, Ha-8 and Ha-9), 1.61-1.51 (m, 2H, Hb-8 and Hb-9); ${ }^{13} \mathrm{C}-\mathrm{NMR}\left(50 \mathrm{MHz}, \mathrm{CDCl}_{3}\right): \delta=138.4,138.3$, 138.2 (s, 3C, C-Ar), 128.2-127.5 (d, 15C, C-Ar), 85.4 (d, C-3), 81.6 (d, C-4), 73.2 (t, C-Bn), 71.4, 71.1, 71.0 (t, 3C, C-Bn and C-6), 69.7 (d, C-2), $62.6(\mathrm{t}, \mathrm{C}-10), 56.9$ (t, C-5), $56.0(\mathrm{t}, \mathrm{C}-7), 31.9,26.0(\mathrm{t}, 2 \mathrm{C}, \mathrm{C}-8$ and C-9); IR $\left(\mathrm{CDCl}_{3}\right): v=3392,3066,3032,2925,2864,1496,1453,1365,1206,1100 \mathrm{~cm}^{-1}$; MS (ESI): $\mathrm{m} / z$ calcd (\%) for $\mathrm{C}_{30} \mathrm{H}_{37} \mathrm{NO}_{4}+\mathrm{H}^{+} 476.27[\mathrm{M}+\mathrm{H}]^{+}$; found: 476.60; elemental analysis calcd (\%) for $\mathrm{C}_{30} \mathrm{H}_{37} \mathrm{NO}_{4}$ (475.62): C 75.76, H 7.84, N 2.94; found: C 75.74, H 7.82, N 2.93.

\subsubsection{Synthesis of Compounds $2123 \alpha / \beta$}

General procedure for the glycosylation reaction: A solution of glucopyranosyl tricholoroacetimidate 18 (1.65 equiv.) and alcohol (1 equiv.) in dry diethyl ether or dry $\mathrm{CH}_{2} \mathrm{Cl}_{2}$, was stirred for $10 \mathrm{~min}$ at room temperature under nitrogen atmosphere in the presence of $3 \AA$ molecular sieves. After cooling to $-20{ }^{\circ} \mathrm{C}$ trimethylsilyl trifluoromethanesulfonate (1.5 equiv.) was added and the mixture was stirred for $1 \mathrm{~h}$; letting the temperature to rise. The mixture was washed with a saturated $\mathrm{Na}_{2} \mathrm{CO}_{3}$ solution, dried over $\mathrm{Na}_{2} \mathrm{SO}_{4}$, filtered and concentrated to dryness. The crude was purified by flash column chromatography on silica gel to afford the pure glycosylated compound as $\alpha, \beta$ mixture.

$21 \alpha, \beta$. Obtained as a brown oil in $50 \%\left(\mathrm{Et}_{2} \mathrm{O}\right), 76 \%\left(\mathrm{CH}_{2} \mathrm{Cl}_{2}\right), 23 \%\left(\mathrm{CH}_{3} \mathrm{CN}\right)$ yields on $0.22-0.34 \mathrm{mmol}$ of alcohol 15. $R_{\mathrm{f}}=0.40$ (EP:AcOEt 4:1); ${ }^{1} \mathrm{H}-\mathrm{NMR}\left(400 \mathrm{MHz}, \mathrm{CDCl}_{3}\right): \delta=7.34-7.05(\mathrm{~m}, 70 \mathrm{H}, \mathrm{H}-\mathrm{Ar})$, $4.98-4.26(\mathrm{~m}, 30 \mathrm{H}, \mathrm{H}-\mathrm{Bn}$ and $\mathrm{OCHO}), 3.97(\mathrm{t}, J=9.3 \mathrm{~Hz}, 2 \mathrm{H}), 3.89-3.84(\mathrm{~m}, 4 \mathrm{H}), 3.78-3.42(\mathrm{~m}, 18 \mathrm{H})$, $3.23-3.12(\mathrm{~m}, 4 \mathrm{H}), 2.82-2.78(\mathrm{~m}, 2 \mathrm{H}), 2.71-2.63(\mathrm{~m}, 4 \mathrm{H})$.

$22 \alpha, \beta$. Obtained as a brown oil in $36 \%\left(\mathrm{Et}_{2} \mathrm{O}\right), 39 \%\left(\mathrm{CH}_{2} \mathrm{Cl}_{2}\right)$ yields on $0.27-0.31 \mathrm{mmol}$ of alcohol 16 . $R_{\mathrm{f}}=0.42$ (EP:AcOEt 4:1); ${ }^{1} \mathrm{H}-\mathrm{NMR}\left(400 \mathrm{MHz}, \mathrm{CDCl}_{3}\right): \delta=7.39-7.15(\mathrm{~m}, 70 \mathrm{H}, \mathrm{H}-\mathrm{Ar}), 5.02-4.41(\mathrm{~m}$, $30 \mathrm{H}, \mathrm{H}-\mathrm{Bn}$ and $\mathrm{OCHO}), 4.03-3.99(\mathrm{~m}, 2 \mathrm{H}), 3.95-3.91(\mathrm{~m}, 4 \mathrm{H}), 3.81-3.44(\mathrm{~m}, 18 \mathrm{H}), 3.24-3.21(\mathrm{~m}, 2 \mathrm{H})$, 3.05-2.93 (m, 2H), 2.79-2.73 (m, 2H), 2.63-2.57 (m, 2H), 2.51-2.45 (m, 2H), 1.98-1.84 (m, 4H).

$23 \alpha, \beta$. Obtained as a brown oil in $66 \%\left(\mathrm{Et}_{2} \mathrm{O}\right), 50 \%\left(\mathrm{CH}_{2} \mathrm{Cl}_{2}\right)$ yields on $0.21-0.33 \mathrm{mmol}$ of alcohol 17 . $R_{\mathrm{f}}=0.45$ (EP:AcOEt 4:1); ${ }^{1} \mathrm{H}-\mathrm{NMR}\left(400 \mathrm{MHz}, \mathrm{CDCl}_{3}\right): \delta=7.30-7.05(\mathrm{~m}, 70 \mathrm{H}, \mathrm{H}-\mathrm{Ar}), 4.92-4.30(\mathrm{~m}, 30 \mathrm{H}$, $\mathrm{H}-\mathrm{Bn}$ and $\mathrm{OCHO}), 3.91(\mathrm{t}, J=9.3 \mathrm{~Hz}, 2 \mathrm{H}), 3.83-3.79(\mathrm{~m}, 4 \mathrm{H}), 3.70-3.31(\mathrm{~m}, 18 \mathrm{H}), 3.13-3.11(\mathrm{~m}, 2 \mathrm{H})$, 2.81-2.74 (m, 2H), 2.65-2.62 (m, 2H), $2.45(\mathrm{dd}, J=10.3,4.9 \mathrm{~Hz}, 2 \mathrm{H}), 2.30-2.24(\mathrm{~m}, 2 \mathrm{H}), 1.56-1.43(\mathrm{~m}, 8 \mathrm{H})$.

\subsubsection{Synthesis of Peracetylated Compounds 24-26}

General procedure for the synthesis of peracetylated derivatives: To a solution of the $\alpha, \beta$ mixture of benzylated derivatives 21-23 (1 equiv.) in 10-20 mL of methanol, $10 \% \mathrm{Pd} / \mathrm{C}(50 \% w / w)$ and $37 \% \mathrm{HCl}$ were added under nitrogen atmosphere, then the mixture was stirred under hydrogen atmosphere at room temperature for $24 \mathrm{~h}$. After that an ${ }^{1} \mathrm{H}-\mathrm{NMR}$ analysis showed the disappearance of the starting material, the mixture was filtered through Celite ${ }^{\circledR}$ and the solvent was removed under reduced pressure. The crude yellow oil was dissolved in pyridine $(2-6 \mathrm{~mL})$ and acetic anhydride $(1-2 \mathrm{~mL})$ was added. The solution was stirred at room temperature overnight. Then, after concentration under 
reduced pressure, the $\alpha$ - and $\beta$-isomers were separated by flash column chromatography on silica gel affording pure peracetylated $\mathbf{2 4 - 2 6} \alpha$ (23-42\% yields over two steps) and $\mathbf{2 4 - 2 6 \beta}$ (3-12\% yields over two steps).

Compound $24 \alpha$. Obtained as a yellow oil in $23 \%$ yield starting from $0.26 \mathrm{mmol}$ of $21 \alpha, \beta . R_{\mathrm{f}}=0.32$ $\left(\mathrm{Et}_{2} \mathrm{O}: \mathrm{CH}_{2} \mathrm{Cl}_{2} 2: 1\right) ;[\alpha]_{\mathrm{D}}^{22}=+61.3\left(c=0.39\right.$ in $\left.\mathrm{CHCl}_{3}\right) ;{ }^{1} \mathrm{H}-\mathrm{NMR}\left(400 \mathrm{MHz}, \mathrm{CDCl}_{3}\right): \delta=5.42(\mathrm{t}, J=9.8 \mathrm{~Hz}$, $\left.1 \mathrm{H}, \mathrm{H}-3^{\prime}\right), 5.09\left(\mathrm{~d}, J=3.9 \mathrm{~Hz}, 1 \mathrm{H}, \mathrm{H}-1^{\prime}\right), 5.06-5.01(\mathrm{~m}, 3 \mathrm{H}, \mathrm{H}-3, \mathrm{H}-4$ and H-4' $), 4.85(\mathrm{dd}, J=10.3,3.9 \mathrm{~Hz}$, $\left.1 \mathrm{H}, \mathrm{H}-2^{\prime}\right), 4.24\left(\mathrm{dd}, J=12.2,4.4 \mathrm{~Hz}, 1 \mathrm{H}, \mathrm{Ha}-6^{\prime}\right), 4.20(\mathrm{dd}, J=11.2,5.9 \mathrm{~Hz}, 1 \mathrm{H}, \mathrm{Ha}-6), 4.10(\mathrm{dd}, J=11.2$, $4.9 \mathrm{~Hz}, 1 \mathrm{H}, \mathrm{Hb}-6), 4.07$ (dd, $\left.J=12.2,1.9 \mathrm{~Hz}, 1 \mathrm{H}, \mathrm{Hb}-6^{\prime}\right), 3.97\left(\mathrm{ddd}, J=10.3,4.4,2.0 \mathrm{~Hz}, 1 \mathrm{H}, \mathrm{H}-5^{\prime}\right.$ ), $3.74(\mathrm{dt}, J=11.3,5.6 \mathrm{~Hz}, 1 \mathrm{H}, \mathrm{Ha}-8), 3.57(\mathrm{dt}, J=11.3,5.9 \mathrm{~Hz}, 1 \mathrm{H}, \mathrm{Hb}-8), 3.18(\mathrm{~d}, J=11.2 \mathrm{~Hz}, 1 \mathrm{H}, \mathrm{Ha}-5)$, $3.03(\mathrm{dt}, J=12.7,6.4 \mathrm{~Hz}, 1 \mathrm{H}, \mathrm{Ha}-7), 2.89(\mathrm{dd}, J=11.2,5.3 \mathrm{~Hz}, 1 \mathrm{H}, \mathrm{Hb}-5), 2.82(\mathrm{q}, J=4.9 \mathrm{~Hz}, 1 \mathrm{H}, \mathrm{H}-2)$, $2.68(\mathrm{dt}, J=12.7,5.9 \mathrm{~Hz}, 1 \mathrm{H}, \mathrm{Hb}-7), 2.07$ (s, 3H, OAc), 2.06-2.05 (m, 12H, OAc), 2.00 (s, 3H, OAc), 1.99 (s, 3H, OAc); ${ }^{13} \mathrm{C}-\mathrm{NMR}\left(50 \mathrm{MHz}, \mathrm{CDCl}_{3}\right): \delta=170.6,170.4,170.1,170.0,169.9,169.5,169.4(\mathrm{~s}, 7 \mathrm{C}$, $\mathrm{C}=\mathrm{O}), 95.7\left(\mathrm{~d}, \mathrm{C}-1^{\prime}\right), 78.7(\mathrm{~d}, \mathrm{C}-4), 76.3(\mathrm{~d}, \mathrm{C}-3), 70.8\left(\mathrm{~d}, \mathrm{C}-2^{\prime}\right), 70.1\left(\mathrm{~d}, \mathrm{C}-3^{\prime}\right), 68.7\left(\mathrm{~d}, \mathrm{C}-4^{\prime}\right), 67.8(\mathrm{~d}, \mathrm{C}-2)$, $67.5\left(\mathrm{~d}, \mathrm{C}-5^{\prime}\right), 66.6(\mathrm{t}, \mathrm{C}-8), 63.2(\mathrm{t}, \mathrm{C}-6), 62.0\left(\mathrm{t}, \mathrm{C}-6^{\prime}\right), 58.0(\mathrm{t}, \mathrm{C}-5), 53.2(\mathrm{t}, \mathrm{C}-7), 20.8,20.7,20.6,20.5(\mathrm{q}$, 7C, $\left.\mathrm{CH}_{3}\right)$; IR $\left(\mathrm{CDCl}_{3}\right): v=2958,2827,2259,1743,1454,1369,1230,1036 \mathrm{~cm}^{-1}$; MS (ESI): $\mathrm{m} / z$ calcd (\%) for $\mathrm{C}_{27} \mathrm{H}_{39} \mathrm{NO}_{16}+\mathrm{Na}^{+} 656.23[\mathrm{M}+\mathrm{Na}]^{+}$; found: 656.33; elemental analysis calcd (\%) for $\mathrm{C}_{27} \mathrm{H}_{39} \mathrm{NO}_{16}$ (633.60): C 51.18, H 6.20, N 2.21; found: C 51.15, H 6.18, N 2.20. Compound 24 $\beta$. Obtained as a yellow oil in $3 \%$ yield starting from $0.26 \mathrm{mmol}$ of $21 \alpha, \beta . R_{\mathrm{f}}=0.23\left(\mathrm{Et}_{2} \mathrm{O}: \mathrm{CH}_{2} \mathrm{Cl}_{2} 2: 1\right) ;[\alpha]_{\mathrm{D}}^{22}=-4.3(c=0.47$ in $\left.\mathrm{CHCl}_{3}\right) ;{ }^{1} \mathrm{H}-\mathrm{NMR}\left(400 \mathrm{MHz}, \mathrm{CDCl}_{3}\right): \delta=5.19\left(\mathrm{t}, J=9.3 \mathrm{~Hz}, 1 \mathrm{H}, \mathrm{H}-3^{\prime}\right), 5.10-5.02\left(\mathrm{~m}, 3 \mathrm{H}, \mathrm{H}-3, \mathrm{H}-4, \mathrm{H}-4^{\prime}\right)$, $4.98\left(\mathrm{dd}, J=9.2,8.3 \mathrm{~Hz}, 1 \mathrm{H}, \mathrm{H}-2^{\prime}\right), 4.52\left(\mathrm{~d}, J=8.3 \mathrm{~Hz}, 1 \mathrm{H}, \mathrm{H}-1^{\prime}\right), 4.25\left(\mathrm{dd}, J=12.2,4.4 \mathrm{~Hz}, 1 \mathrm{H}, \mathrm{Ha}-6^{\prime}\right)$, $4.22(\mathrm{dd}, J=11.2,5.8 \mathrm{~Hz}, 1 \mathrm{H}, \mathrm{Ha}-6), 4.14\left(\mathrm{dd}, J=12.2,2.4 \mathrm{~Hz}, 1 \mathrm{H}, \mathrm{Hb}-6^{\prime}\right), 4.09(\mathrm{dd}, J=11.2,4.4 \mathrm{~Hz}, 1 \mathrm{H}$, Hb-6), $3.97(\mathrm{dt}, J=9.8,4.9 \mathrm{~Hz}, 1 \mathrm{H}, \mathrm{Ha}-8), 3.68\left(\mathrm{ddd}, J=9.7,4.9,2.4 \mathrm{~Hz}, 1 \mathrm{H}, \mathrm{H}-5^{\prime}\right), 3.61(\mathrm{ddd}, J=10.2,7.3$, $4.4 \mathrm{~Hz}, 1 \mathrm{H}, \mathrm{Hb}-8), 3.12(\mathrm{~d}, J=11.7 \mathrm{~Hz}, 1 \mathrm{H}, \mathrm{Ha}-5), 3.00(\mathrm{dt}, J=13.2,4.9 \mathrm{~Hz}, 1 \mathrm{H}, \mathrm{Ha}-7), 2.84(\mathrm{dd}, J=11.7$, $5.4 \mathrm{~Hz}, 1 \mathrm{H}, \mathrm{Hb}-5), 2.80-2.76$ (m, 1H, H-2), 2.66 (ddd, J = 13.2, 7.8, 4.4 Hz, 1H, Hb-7), 2.08 (s, 3H, OAc), 2.07 (m, 6H, OAc), 2.06 (s, 3H, OAc), 2.03 (s, 3H, OAc), 2.01 (s, 3H, OAc), 1.99 (s, 3H, OAc); ${ }^{13} \mathrm{C}-\mathrm{NMR}$ $\left(100 \mathrm{MHz} \mathrm{CDCl}_{3}\right): \delta=170.8,170.6,170.2,170.1,169.5,169.4,169.2(\mathrm{~s}, 7 \mathrm{C}, \mathrm{C}=\mathrm{O}), 100.5\left(\mathrm{~d}, \mathrm{C}-1^{\prime}\right), 78.5(\mathrm{~d}$, C-4), $76.5(\mathrm{~d}, \mathrm{C}-3), 72.8\left(\mathrm{~d}, \mathrm{C}-3^{\prime}\right), 71.9\left(\mathrm{~d}, \mathrm{C}-5^{\prime}\right), 71.2\left(\mathrm{~d}, \mathrm{C}-2^{\prime}\right), 68.8(\mathrm{t}, \mathrm{C}-8), 68.4\left(\mathrm{~d}, \mathrm{C}-4^{\prime}\right), 68.0(\mathrm{~d}, \mathrm{C}-2)$, $62.9(\mathrm{t}, \mathrm{C}-6), 61.9\left(\mathrm{t}, \mathrm{C}-6^{\prime}\right), 58.3(\mathrm{t}, \mathrm{C}-5), 53.3(\mathrm{t}, \mathrm{C}-7), 21.0,20.9,20.8,20.7,20.6\left(\mathrm{q}, 7 \mathrm{C}, \mathrm{CH}_{3}\right) ; \mathrm{IR}\left(\mathrm{CDCl}_{3}\right)$ : $v=2958,2827,2259,1747,1454,1369,1225,1036 \mathrm{~cm}^{-1}$; MS (ESI): $\mathrm{m} / z$ calcd (\%) for $\mathrm{C}_{27} \mathrm{H}_{39} \mathrm{NO}_{16}+\mathrm{Na}^{+}$ $656.23[\mathrm{M}+\mathrm{Na}]^{+}$; found: 656.42; elemental analysis calcd (\%) for $\mathrm{C}_{27} \mathrm{H}_{39} \mathrm{NO}_{16}$ (633.60): $\mathrm{C} 51.18, \mathrm{H}$ 6.20, N 2.21; found: C 51.15, H 6.18, N 2.19.

Compound $25 \alpha$. Obtained as a yellow oil in $42 \%$ yield starting from $0.12 \mathrm{mmol}$ of $22 \alpha, \beta . R_{\mathrm{f}}=0.34$ $\left(\mathrm{Et}_{2} \mathrm{O}: \mathrm{CH}_{2} \mathrm{Cl}_{2} 3: 1\right) ;[\alpha]_{\mathrm{D}}^{23}=+48.6\left(c=0.42\right.$ in $\left.\mathrm{CHCl}_{3}\right) ;{ }^{1} \mathrm{H}-\mathrm{NMR}\left(400 \mathrm{MHz}, \mathrm{CDCl}_{3}\right): \delta=5.44(\mathrm{t}, J=9.8 \mathrm{~Hz}$, $\left.1 \mathrm{H}, \mathrm{H}-3^{\prime}\right), 5.06-5.01\left(\mathrm{~m}, 4 \mathrm{H}, \mathrm{H}-3, \mathrm{H}-4, \mathrm{H}^{-} \mathbf{1}^{\prime}\right.$ and H-4' $), 4.86\left(\mathrm{dd}, J=10.2,3.4 \mathrm{~Hz}, 1 \mathrm{H}, \mathrm{H}-2^{\prime}\right), 4.23(\mathrm{dd}$, $\left.J=12.2,4.4 \mathrm{~Hz}, 1 \mathrm{H}, \mathrm{Ha}-6^{\prime}\right), 4.15(\mathrm{dd}, J=11.2,6.3 \mathrm{~Hz}, 1 \mathrm{H}, \mathrm{Ha}-6), 4.10(\mathrm{dd}, J=11.2,4.9 \mathrm{~Hz}, 1 \mathrm{H}, \mathrm{Hb}-6)$, $4.07\left(\mathrm{dd}, J=12.2,2.4 \mathrm{~Hz}, 1 \mathrm{H}, \mathrm{Hb}-6^{\prime}\right), 3.97\left(\mathrm{ddd}, J=10.3,4.4,2.4 \mathrm{~Hz}, 1 \mathrm{H}, \mathrm{H}-5^{\prime}\right), 3.71(\mathrm{dt}, J=10.3,6.4 \mathrm{~Hz}$, 1H, Ha-9), $3.44(\mathrm{dt}, J=10.3,6.6 \mathrm{~Hz}, 1 \mathrm{H}, \mathrm{Hb}-9), 3.12(\mathrm{~d}, J=11.2 \mathrm{~Hz}, 1 \mathrm{H}, \mathrm{Ha}-5), 2.87(\mathrm{dt}, J=12.2,7.9 \mathrm{~Hz}$, 1H, Ha-7), $2.76(\mathrm{dd}, J=11.2,4.9 \mathrm{~Hz}, 1 \mathrm{H}, \mathrm{Hb}-5), 2.75-2.71(\mathrm{~m}, 1 \mathrm{H}, \mathrm{H}-2), 2.45(\mathrm{dt}, J=12.2,6.4 \mathrm{~Hz}, 1 \mathrm{H}$, Hb-7), 2.08 (m, 6H, OAc), 2.06 (m, 6H, OAc), 2.04 (s, 3H, OAc), 2.01 (s, 3H, OAc), 1.99 (s, 3H, OAc), 1.77 (quint, $J=6.9 \mathrm{~Hz}, 2 \mathrm{H}, \mathrm{H}-8) ;{ }^{13} \mathrm{C}-\mathrm{NMR}\left(100 \mathrm{MHz}, \mathrm{CDCl}_{3}\right): \delta=170.6,170.4,170.3,170.2,170.1,169.9$, $169.4(\mathrm{~s}, 7 \mathrm{C}, \mathrm{C}=\mathrm{O}), 95.8\left(\mathrm{~d}, \mathrm{C}-\mathrm{1}^{\prime}\right), 78.9(\mathrm{~d}, \mathrm{C}-4), 76.3(\mathrm{~d}, \mathrm{C}-3), 70.9\left(\mathrm{~d}, \mathrm{C}-2^{\prime}\right), 70.3\left(\mathrm{~d}, \mathrm{C}-3^{\prime}\right), 68.8\left(\mathrm{~d}, \mathrm{C}-4^{\prime}\right)$, $67.8(\mathrm{~d}, \mathrm{C}-2), 67.3\left(\mathrm{~d}, \mathrm{C}-5^{\prime}\right), 66.3(\mathrm{t}, \mathrm{C}-9), 63.2(\mathrm{t}, \mathrm{C}-6), 62.0\left(\mathrm{t}, \mathrm{C}-6^{\prime}\right), 57.4(\mathrm{t}, \mathrm{C}-5), 51.2(\mathrm{t}, \mathrm{C}-7), 28.0(\mathrm{t}, \mathrm{C}-8)$, 20.8, 20.7, 20.6, 20.5 (q, 7C, $\left.\mathrm{CH}_{3}\right)$; IR $\left(\mathrm{CDCl}_{3}\right): v=2952,2872,2261,1743,1455,1374,1234,1039 \mathrm{~cm}^{-1}$; MS (ESI): $\mathrm{m} / \mathrm{z}$ calcd (\%) for $\mathrm{C}_{28} \mathrm{H}_{41} \mathrm{NO}_{16}+\mathrm{Na}^{+} 670.24$ [M + Na] $]^{+}$; found: 669.72; elemental analysis calcd (\%) for $\mathrm{C}_{28} \mathrm{H}_{41} \mathrm{NO}_{16}$ (647.62): C 51.93, H 6.38, N 2.16; found: C 51.92, H 6.29, N 2.15. Compound $25 \beta$. Obtained as a yellow oil in $12 \%$ yield starting from $0.12 \mathrm{mmol}$ of $22 \alpha, \beta . R_{\mathrm{f}}=0.26\left(\mathrm{Et}_{2} \mathrm{O}: \mathrm{CH}_{2} \mathrm{Cl}_{2}\right.$ 3:1); $[\alpha]_{\mathrm{D}}^{21}=-24.2\left(c=0.12\right.$ in $\left.\mathrm{CHCl}_{3}\right) ;{ }^{1} \mathrm{H}-\mathrm{NMR}\left(400 \mathrm{MHz}, \mathrm{CDCl}_{3}\right): \delta=5.19\left(\mathrm{t}, J=9.7 \mathrm{~Hz}, 1 \mathrm{H}, \mathrm{H}-3^{\prime}\right)$, $5.07\left(\mathrm{t}, J=9.7 \mathrm{~Hz}, 1 \mathrm{H}, \mathrm{H}-4^{\prime}\right), 5.07-5.03\left(\mathrm{~m}, 2 \mathrm{H}, \mathrm{H}-3\right.$ and H-4), $4.96\left(\mathrm{dd}, J=9.7,7.8 \mathrm{~Hz}, 1 \mathrm{H}, \mathrm{H}-2^{\prime}\right), 4.48(\mathrm{~d}$, $\left.J=7.8 \mathrm{~Hz}, 1 \mathrm{H}, \mathrm{H}-1^{\prime}\right), 4.25\left(\mathrm{dd}, J=12.2,4.9 \mathrm{~Hz}, 1 \mathrm{H}, \mathrm{Ha}-6^{\prime}\right), 4.15-4.12(\mathrm{~m}, 2 \mathrm{H}, \mathrm{H}-6), 4.13(\mathrm{dd}, J=12.2$, 
$\left.2.4 \mathrm{~Hz}, 1 \mathrm{H}, \mathrm{Hb}-6^{\prime}\right), 3.87$ (dt, $\left.J=9.4,6.4 \mathrm{~Hz}, 1 \mathrm{H}, \mathrm{Ha}-9\right), 3.68\left(\mathrm{ddd}, J=10.2,4.4,2.4 \mathrm{~Hz}, 1 \mathrm{H}, \mathrm{H}-5^{\prime}\right), 3.53$ (dt, $J=9.7,6.4 \mathrm{~Hz}, 1 \mathrm{H}, \mathrm{Hb}-9), 3.11(\mathrm{~d}, J=10.7 \mathrm{~Hz}, 1 \mathrm{H}, \mathrm{Ha}-5), 2.83(\mathrm{dt}, J=12.2,7.8 \mathrm{~Hz}, 1 \mathrm{H}, \mathrm{Ha}-7), 2.76-2.72$ (m, 1H, H-2), 2.75 (dd, J = 11.2, 4.8 Hz, 1H, Hb-5), 2.43 (dt, $J=12.2,6.4 \mathrm{~Hz}, 1 \mathrm{H}, \mathrm{Hb}-7), 2.08$ (m, 6H, OAc), 2.07 (s, 3H, OAc), 2.06 (s, 3H, OAc), 2.04 (s, 3H, OAc), 2.01 (s, 3H, OAc), 1.99 (s, 3H, OAc), 1.74 (quint, $J=6.8 \mathrm{~Hz}, 2 \mathrm{H}, \mathrm{H}-8) ;{ }^{13} \mathrm{C}-\mathrm{NMR}\left(100 \mathrm{MHz}, \mathrm{CDCl}_{3}\right): \delta=170.8,170.6,170.2,170.0,169.5,169.3,169.1(\mathrm{~s}, 7 \mathrm{C}$, $\mathrm{C}=\mathrm{O}), 100.7\left(\mathrm{~d}, \mathrm{C}-1^{\prime}\right), 78.8(\mathrm{~d}, \mathrm{C}-4), 76.3(\mathrm{~d}, \mathrm{C}-3), 72.8\left(\mathrm{~d}, \mathrm{C}-3^{\prime}\right), 71.8\left(\mathrm{~d}, \mathrm{C}-5^{\prime}\right), 71.3\left(\mathrm{~d}, \mathrm{C}-2^{\prime}\right), 68.5\left(\mathrm{~d}, \mathrm{C}-4^{\prime}\right)$, $67.8(\mathrm{t}, \mathrm{C}-9), 67.7(\mathrm{~d}, \mathrm{C}-2), 63.3(\mathrm{t}, \mathrm{C}-6), 62.0\left(\mathrm{t}, \mathrm{C}-6^{\prime}\right), 57.4(\mathrm{t}, \mathrm{C}-5), 51.3(\mathrm{t}, \mathrm{C}-7), 28.1(\mathrm{t}, \mathrm{C}-8), 21.0,20.9$, 20.8, 20.6, 20.5 (q, 7C, $\left.\mathrm{CH}_{3}\right)$; IR $\left(\mathrm{CDCl}_{3}\right): v=2954,2882,2261,1743,1376,1234,1039 \mathrm{~cm}^{-1}$; MS (ESI): $m / z$ calcd (\%) for $\mathrm{C}_{28} \mathrm{H}_{41} \mathrm{NO}_{16}+\mathrm{Na}^{+} 670.24[\mathrm{M}+\mathrm{Na}]^{+}$; found: 670.36 ; elemental analysis calcd $(\%)$ for $\mathrm{C}_{28} \mathrm{H}_{41} \mathrm{NO}_{16}$ (647.62): C 51.93, H 6.38, N 2.16; found: C 51.90, H 6.32, N 2.14.

Compound 26 $\alpha$. Obtained as a yellow oil in $37 \%$ yield starting from $0.095 \mathrm{mmol}$ of $23 \alpha, \beta . R_{\mathrm{f}}=0.25$ $\left(\mathrm{Et}_{2} \mathrm{O}: \mathrm{CH}_{2} \mathrm{Cl}_{2} 4: 1\right) ;[\alpha]_{\mathrm{D}}^{23}=+44.6\left(c=0.74\right.$ in $\left.\mathrm{CHCl}_{3}\right) ;{ }^{1} \mathrm{H}-\mathrm{NMR}\left(400 \mathrm{MHz}, \mathrm{CDCl}_{3}\right): \delta=5.46(\mathrm{t}, J=9.8 \mathrm{~Hz}$, $\left.1 \mathrm{H}, \mathrm{H}-3^{\prime}\right), 5.07-5.02\left(\mathrm{~m}, 4 \mathrm{H}, \mathrm{H}-3, \mathrm{H}-4, \mathrm{H}-1^{\prime}\right.$ and H-4' $), 4.84\left(\mathrm{dd}, J=10.2,3.9 \mathrm{~Hz}, 1 \mathrm{H}, \mathrm{H}-2^{\prime}\right), 4.25$ (dd, $\left.J=12.7,4.4 \mathrm{~Hz}, 1 \mathrm{H}, \mathrm{Ha}-6^{\prime}\right), 4.19(\mathrm{dd}, J=11.7,6.4 \mathrm{~Hz}, 1 \mathrm{H}, \mathrm{Ha}-6), 4.12(\mathrm{dd}, J=11.7,4.8 \mathrm{~Hz}, 1 \mathrm{H}, \mathrm{Hb}-6)$, $4.08\left(\mathrm{dd}, J=12.7,2.4 \mathrm{~Hz}, 1 \mathrm{H}, \mathrm{Hb}-6^{\prime}\right), 3.99\left(\mathrm{ddd}, J=10.2,4.4,2.4 \mathrm{~Hz}, 1 \mathrm{H}, \mathrm{H}-5^{\prime}\right), 3.68(\mathrm{dt}, J=9.7,6.3 \mathrm{~Hz}$, $1 \mathrm{H}, \mathrm{Ha}-10), 3.42(\mathrm{dt}, J=9.7,6.8 \mathrm{~Hz}, 1 \mathrm{H}, \mathrm{Hb}-10), 3.14(\mathrm{~d}, J=11.2 \mathrm{~Hz}, 1 \mathrm{H}, \mathrm{Ha}-5), 2.80(\mathrm{dt}, J=11.7,7.8 \mathrm{~Hz}$, 1H, Ha-7), 2.73 (dd, J = 10.8, 4.9 Hz, 1H, Hb-5), 2.72-2.70 (m, 1H, H-2), 2.40-2.34 (m, 1H, Hb-7), 2.08 (m, 6H, OAc), 2.07 (s, 3H, OAc), 2.06 (s, 3H, OAc), 2.05 (s, 3H, OAc), 2.02 (s, 3H, OAc), 2.00 (s, 3H, OAc), 1.69-1.48 (m, 4H, H-8 and H-9); ${ }^{13} \mathrm{C}-\mathrm{NMR}\left(50 \mathrm{MHz}, \mathrm{CDCl}_{3}\right): \delta=170.7,170.6,170.5,170.2,170.1,170.0$, $169.5(\mathrm{~s}, 7 \mathrm{C}, \mathrm{C}=\mathrm{O}), 95.8\left(\mathrm{~d}, \mathrm{C}-1^{\prime}\right), 78.9(\mathrm{~d}, \mathrm{C}-4), 76.3(\mathrm{~d}, \mathrm{C}-3), 71.0\left(\mathrm{~d}, \mathrm{C}-2^{\prime}\right), 70.3\left(\mathrm{~d}, \mathrm{C}-3^{\prime}\right), 68.8\left(\mathrm{~d}, \mathrm{C}-4^{\prime}\right)$, $68.4(\mathrm{t}, \mathrm{C}-10), 67.8(\mathrm{~d}, \mathrm{C}-2), 67.3\left(\mathrm{~d}, \mathrm{C}-5^{\prime}\right), 63.2(\mathrm{t}, \mathrm{C}-6), 62.0\left(\mathrm{t}, \mathrm{C}-6^{\prime}\right), 57.3(\mathrm{t}, \mathrm{C}-5), 54.4(\mathrm{t}, \mathrm{C}-7), 27.1(\mathrm{t}$, C-9), 24.7 (t, C-8), 20.9, 20.7, 20.5 (q, 7C, $\left.\mathrm{CH}_{3}\right)$; IR $\left(\mathrm{CDCl}_{3}\right): v=2953,2872,2260,1742,1455,1373,1233$, $1038 \mathrm{~cm}^{-1}$; MS (ESI): $m / z$ calcd (\%) for $\mathrm{C}_{29} \mathrm{H}_{43} \mathrm{NO}_{16}+\mathrm{Na}^{+} 684.26[\mathrm{M}+\mathrm{Na}]^{+}$; found: 683.63; elemental analysis calcd (\%) for $\mathrm{C}_{29} \mathrm{H}_{43} \mathrm{NO}_{16}$ (661.65): C 52.64, H 6.55, N 2.12; found: C 52.61, H 6.59, N 2.17.

Compound 26 $\beta$. Obtained as a yellow oil in $9 \%$ yield starting from $0.095 \mathrm{mmol}$ of $23 \alpha, \beta . R_{\mathrm{f}}=0.20$ $\left(\mathrm{Et}_{2} \mathrm{O}: \mathrm{CH}_{2} \mathrm{Cl}_{2} 4: 1\right) ;[\alpha]_{\mathrm{D}}^{23}=-35.2\left(c=0.17\right.$ in $\left.\mathrm{CHCl}_{3}\right) ;{ }^{1} \mathrm{H}-\mathrm{NMR}\left(400 \mathrm{MHz}, \mathrm{CDCl}_{3}\right): \delta=5.19(\mathrm{t}, J=9.8 \mathrm{~Hz}$, $\left.1 \mathrm{H}, \mathrm{H}-3^{\prime}\right), 5.07\left(\mathrm{t}, J=9.8 \mathrm{~Hz}, 1 \mathrm{H}, \mathrm{H}-4^{\prime}\right), 5.07-5.03(\mathrm{~m}, 2 \mathrm{H}, \mathrm{H}-3$ and $\mathrm{H}-4), 4.97(\mathrm{dd}, J=9.8,7.8 \mathrm{~Hz}, 1 \mathrm{H}$, H-2' $), 4.48\left(\mathrm{~d}, J=7.8 \mathrm{~Hz}, 1 \mathrm{H}, \mathrm{H}-1^{\prime}\right), 4.25\left(\mathrm{dd}, J=12.2,4.4 \mathrm{~Hz}, 1 \mathrm{H}, \mathrm{Ha}-6^{\prime}\right), 4.19(\mathrm{dd}, J=11.2,6.3 \mathrm{~Hz}$, $1 \mathrm{H}$, Ha-6), $4.12\left(\mathrm{dd}, J=12.2,2.4 \mathrm{~Hz}, 1 \mathrm{H}, \mathrm{Hb}-6^{\prime}\right), 4.10(\mathrm{dd}, J=11.2,4.9 \mathrm{~Hz}, 1 \mathrm{H}, \mathrm{Hb}-6), 3.87(\mathrm{dt}, J=9.8$, $5.9 \mathrm{~Hz}, 1 \mathrm{H}, \mathrm{Ha}-10), 3.68\left(\mathrm{ddd}, J=9.8,4.4,2.4 \mathrm{~Hz}, 1 \mathrm{H}, \mathrm{H}-5^{\prime}\right), 3.47(\mathrm{dt}, J=9.8,6.8 \mathrm{~Hz}, 1 \mathrm{H}, \mathrm{Hb}-10), 3.11$ (d, $J=11.2 \mathrm{~Hz}, 1 \mathrm{H}, \mathrm{Ha}-5), 2.77$ (dt, $J=12.2,7.8 \mathrm{~Hz}, 1 \mathrm{H}, \mathrm{Ha}-7), 2.70(\mathrm{dd}, J=11.2,5.2 \mathrm{~Hz}, 1 \mathrm{H}, \mathrm{Hb}-5), 2.70-2.67$ (m, 1H, H-2), 2.33 (ddd, $J=12.2,8.8,4.4 \mathrm{~Hz}, 1 \mathrm{H}, \mathrm{Hb}-7), 2.07$ (m, 6H, OAc), 2.06 (s, 3H, OAc), 2.03 (s, 3H, OAc), 2.02 (s, 3H, OAc), 2.01 (s, 3H, OAc), 1.99 (s, 3H, OAc), 1.63-1.42 (m, 4H, H-8 and H-9); ${ }^{13} \mathrm{C}-\mathrm{NMR}$ $\left(50 \mathrm{MHz}, \mathrm{CDCl}_{3}\right): \delta=170.7,170.5,170.1,170.0,169.5,169.3,169.1(\mathrm{~s}, 7 \mathrm{C}, \mathrm{C}=\mathrm{O}), 100.7\left(\mathrm{~d}, \mathrm{C}-1^{\prime}\right), 78.9(\mathrm{~d}$, C-4), $76.3(\mathrm{~d}, \mathrm{C}-3), 72.9\left(\mathrm{~d}, \mathrm{C}-3^{\prime}\right), 71.8\left(\mathrm{~d}, \mathrm{C}-5^{\prime}\right), 71.4\left(\mathrm{~d}, \mathrm{C}-2^{\prime}\right), 69.6(\mathrm{t}, \mathrm{C}-10), 68.6\left(\mathrm{~d}, \mathrm{C}-4^{\prime}\right), 67.8(\mathrm{~d}, \mathrm{C}-2)$, $63.1(\mathrm{t}, \mathrm{C}-6), 62.0\left(\mathrm{t}, \mathrm{C}-6^{\prime}\right), 57.2(\mathrm{t}, \mathrm{C}-5), 54.2(\mathrm{t}, \mathrm{C}-7), 27.1(\mathrm{t}, \mathrm{C}-9), 24.3(\mathrm{t}, \mathrm{C}-8), 20.9,20.8,20.7,20.6,20.5$, $20.4\left(\mathrm{q}, 7 \mathrm{C}, \mathrm{CH}_{3}\right)$; IR $\left(\mathrm{CDCl}_{3}\right): v=2957,2886,2816,2261,1743,1454,1373,1233,1039 \mathrm{~cm}^{-1}$; MS (ESI): $m / z$ calcd (\%) for $\mathrm{C}_{29} \mathrm{H}_{43} \mathrm{NO}_{16}+\mathrm{Na}^{+} 684.26[\mathrm{M}+\mathrm{Na}]^{+}$; found: 683.90; elemental analysis calcd (\%) for $\mathrm{C}_{29} \mathrm{H}_{43} \mathrm{NO}_{16}$ (661.65): C 52.64, H 6.55, N 2.12; found: C 52.60, H 6.52, N 2.11.

\subsubsection{Synthesis of Compounds $\mathbf{9 - 1 1}$}

General procedure for the synthesis of the $\alpha, \beta$ mixture of polyhydroxylated pseudodisaccharides: To a $0.01 \mathrm{M}$ solution of the $\alpha, \beta$ mixture of benzylated derivatives $21-23 \alpha, \beta$ in methanol, $\mathrm{Pd} / \mathrm{C}(50 \%$, $w / w)$ and two drops of $37 \% \mathrm{HCl}$ were added under nitrogen atmosphere, then the mixture was stirred under hydrogen atmosphere at room temperature for 1-3 days, until a NMR control showed the disappearance of the starting material. The mixture was then filtered through Celite ${ }^{\circledR}$ and the solvent was removed under reduced pressure affording a crude yellow oil. The $\alpha, \beta$ mixture of free amines was obtained by passing the corresponding hydrochloride salts mixture through a Dowex 50WX8 
ion-exchange resin. Elution with $6 \% \mathrm{NH}_{4} \mathrm{OH}$ afforded the free bases $9 \alpha, \beta, 10 \alpha, \beta$ and $11 \alpha, \beta$ with $32-40 \%$ yields.

Compounds $9 \alpha, \beta$ : The general procedure applied to $144 \mathrm{mg}(0.146 \mathrm{mmol})$ of $21 \alpha, \beta$, stirring the mixture for 3 days, afforded $17 \mathrm{mg}(0.05 \mathrm{mmol})$ of a 2:1 $9 \alpha, \beta$ mixture in $35 \%$ yield.

Compounds $10 \alpha, \beta$ : The general procedure applied to $100 \mathrm{mg}(0.102 \mathrm{mmol})$ of $22 \alpha, \beta$, stirring the mixture for one day, afforded $14 \mathrm{mg}(0.04 \mathrm{mmol})$ of a 1:1 $10 \alpha, \beta$ mixture in $40 \%$ yield.

Compounds $11 \alpha, \beta$ : The general procedure applied to $192 \mathrm{mg}(0.192 \mathrm{mmol})$ of $23 \alpha, \beta$, stirring the mixture for 3 days, afforded $21 \mathrm{mg}(0.06 \mathrm{mmol})$ of a 1.2:1 11 $\alpha, \beta$ mixture in $32 \%$ yield.

General procedure for the synthesis of polyhydroxylated $\alpha$-pseudodisaccharides: A suspension of $\alpha$-peracetylated derivatives 24 $\alpha-26 \alpha$ (1 equiv.) and ion exchange resin Ambersep $900 \mathrm{OH}(600 \mathrm{mg}$ ) in 5-10 mL of methanol was slowly stirred at room temperature for $16 \mathrm{~h}$. After filtration through Celite ${ }^{\circledR}$, the solvent was removed under reduced pressure affording pure $\alpha$-pseudodisaccharides $\mathbf{9} \alpha-\mathbf{1 1} \alpha$ in quantitative yields.

Compound $9 \alpha$. Obtained as a yellow oil in quantitative yield starting from $0.063 \mathrm{mmol}$ of $24 \alpha$. $[\alpha]_{\mathrm{D}}^{27}=+48.4(c=1.11$ in $\mathrm{MeOH}) ;{ }^{1} \mathrm{H}-\mathrm{NMR}\left(400 \mathrm{MHz}, \mathrm{D}_{2} \mathrm{O}\right): \delta=4.72\left(\mathrm{~d}, J=3.9 \mathrm{~Hz}, 1 \mathrm{H}, \mathrm{H}-1^{\prime}\right), 3.93(\mathrm{ddd}$, $J=5.4,2.9,2.4 \mathrm{~Hz}, 1 \mathrm{H}, \mathrm{H}-4), 3.73(\mathrm{dd}, J=4.9,2.9 \mathrm{~Hz}, 1 \mathrm{H}, \mathrm{H}-3), 3.67\left(\mathrm{dd}, J=12.2,2.4 \mathrm{~Hz}, 1 \mathrm{H}, \mathrm{Ha}-6^{\prime}\right)$, 3.67-3.64 (m, 1H, Ha-8), $3.56\left(\mathrm{dd}, J=12.2,5.4 \mathrm{~Hz}, 1 \mathrm{H}, \mathrm{Hb}-6^{\prime}\right), 3.55-3.50\left(\mathrm{~m}, 3 \mathrm{H}, \mathrm{H}-3^{\prime}\right.$ and H-6), 3.47 (ddd, $\left.J=9.7,5.4,2.4 \mathrm{~Hz}, 1 \mathrm{H}, \mathrm{H}-5^{\prime}\right), 3.39(\mathrm{ddd}, J=12.2,8.3,4.4 \mathrm{~Hz}, 1 \mathrm{H}, \mathrm{Hb}-8), 3.34(\mathrm{dd}, J=9.7,3.9 \mathrm{~Hz}, 1 \mathrm{H}$, H-2' $), 3.21\left(\mathrm{t}, J=9.7 \mathrm{~Hz}, 1 \mathrm{H}, \mathrm{H}-4^{\prime}\right), 2.97(\mathrm{ddd}, J=13.2,7.8,4.4 \mathrm{~Hz}, 1 \mathrm{H}, \mathrm{Ha}-7), 2.86(\mathrm{dd}, J=11.3,2.0 \mathrm{~Hz}$, $1 \mathrm{H}, \mathrm{Ha}-5), 2.61(\mathrm{dd}, J=11.3,5.9 \mathrm{~Hz}, 1 \mathrm{H}, \mathrm{Hb}-5), 2.46(\mathrm{dt}, J=13.2,4.4 \mathrm{~Hz}, 1 \mathrm{H}, \mathrm{Hb}-7), 2.38(\mathrm{q}, J=5.4 \mathrm{~Hz}$, 1H, H-2); ${ }^{13} \mathrm{C}-\mathrm{NMR}\left(100 \mathrm{MHz}, \mathrm{D}_{2} \mathrm{O}\right): \delta=98.4\left(\mathrm{~d}, \mathrm{C}-1^{\prime}\right), 78.9(\mathrm{~d}, \mathrm{C}-3), 75.4(\mathrm{~d}, \mathrm{C}-4), 73.1\left(\mathrm{~d}, \mathrm{C}-3^{\prime}\right), 72.0(\mathrm{~d}$, C-5'), $71.9\left(\mathrm{~d}, \mathrm{C}-2^{\prime}\right), 71.4(\mathrm{~d}, \mathrm{C}-2), 69.5\left(\mathrm{~d}, \mathrm{C}-4^{\prime}\right), 65.6(\mathrm{t}, \mathrm{C}-8), 61.1(\mathrm{t}, \mathrm{C}-6), 60.5\left(\mathrm{t}, \mathrm{C}-6^{\prime}\right), 58.6(\mathrm{t}, \mathrm{C}-5), 54.1$ (t, C-7); MS (ESI): $m / z$ calcd (\%) for $\mathrm{C}_{13} \mathrm{H}_{25} \mathrm{NO}_{9}+\mathrm{Na}^{+} 362.15[\mathrm{M}+\mathrm{Na}]^{+}$; found: 361.52; elemental analysis calcd (\%) for $\mathrm{C}_{13} \mathrm{H}_{25} \mathrm{NO}_{9}$ (339.34): $\mathrm{C} 46.01, \mathrm{H}$ 7.43, N 4.13; found: C 45.98, H 7.48, N 4.16.

Compound $10 \alpha$. Obtained as a yellow oil in quantitative yield starting from $0.046 \mathrm{mmol}$ of $25 \alpha$. $[\alpha]_{\mathrm{D}}^{26}=+34.1(c=0.81$ in $\mathrm{MeOH}) ;{ }^{1} \mathrm{H}-\mathrm{NMR}\left(400 \mathrm{MHz}, \mathrm{D}_{2} \mathrm{O}\right): \delta=4.71\left(\mathrm{~d}, J=3.4 \mathrm{~Hz}, 1 \mathrm{H}, \mathrm{H}-\mathrm{1}^{\prime}\right), 3.92(\mathrm{ddd}$, $J=5.4,2.9,2.0 \mathrm{~Hz}, 1 \mathrm{H}, \mathrm{H}-4), 3.73(\mathrm{dd}, J=5.4,2.9 \mathrm{~Hz}, 1 \mathrm{H}, \mathrm{H}-3), 3.66\left(\mathrm{dd}, J=12.2,2.4 \mathrm{~Hz}, 1 \mathrm{H}, \mathrm{Ha}-6^{\prime}\right)$, $3.56\left(\mathrm{dd}, J=12.2,5.4 \mathrm{~Hz}, 1 \mathrm{H}, \mathrm{Hb}-6^{\prime}\right), 3.54-3.50$ (m, 4H, H-3', H-6 and Ha-9), 3.46 (ddd, $J=9.7,5.4$, $\left.2.4 \mathrm{~Hz}, 1 \mathrm{H}, \mathrm{H}-5^{\prime}\right), 3.40-3.36(\mathrm{~m}, 1 \mathrm{H}, \mathrm{Hb}-9), 3.35\left(\mathrm{dd}, J=9.7,3.5 \mathrm{~Hz}, 1 \mathrm{H}, \mathrm{H}-2^{\prime}\right), 3.21(\mathrm{t}, J=9.7 \mathrm{~Hz}, 1 \mathrm{H}$, H-4') $2.82(\mathrm{dd}, J=11.2,1.9 \mathrm{~Hz}, 1 \mathrm{H}, \mathrm{Ha}-5), 2.82-2.76$ (m, 1H, Ha-7), 2.55 (dd, $J=11.2,5.9 \mathrm{~Hz}, 1 \mathrm{H}, \mathrm{Hb}-5)$, $2.35(\mathrm{q}, J=5.4 \mathrm{~Hz}, 1 \mathrm{H}, \mathrm{H}-2), 2.26(\mathrm{ddd}, J=11.7,9.8,5.9 \mathrm{~Hz}, 1 \mathrm{H}, \mathrm{Hb}-7), 1.72-1.60(\mathrm{~m}, 2 \mathrm{H}, \mathrm{H}-8) ;{ }^{13} \mathrm{C}-\mathrm{NMR}$ $\left(100 \mathrm{MHz}, \mathrm{D}_{2} \mathrm{O}\right): \delta=98.0\left(\mathrm{~d}, \mathrm{C}-1^{\prime}\right), 79.0(\mathrm{~d}, \mathrm{C}-3), 75.3(\mathrm{~d}, \mathrm{C}-4), 73.0\left(\mathrm{~d}, \mathrm{C}-3^{\prime}\right), 71.8\left(\mathrm{~d}, \mathrm{C}-5^{\prime}\right), 71.3(\mathrm{~d}, 2 \mathrm{C}$, C-2' and C-2), $69.5\left(\mathrm{~d}, \mathrm{C}-4^{\prime}\right), 66.2(\mathrm{t}, \mathrm{C}-9), 61.2(\mathrm{t}, \mathrm{C}-6), 60.5\left(\mathrm{t}, \mathrm{C}-6^{\prime}\right), 58.2(\mathrm{t}, \mathrm{C}-5), 52.2(\mathrm{t}, \mathrm{C}-7), 26.9(\mathrm{t}$, C-8); MS (ESI): $m / z$ calcd (\%) for $\mathrm{C}_{14} \mathrm{H}_{27} \mathrm{NO}_{9}{ }^{+} 353.17$ [M] ${ }^{+}$; found: 352.96 ; elemental analysis calcd (\%) for $\mathrm{C}_{14} \mathrm{H}_{27} \mathrm{NO}_{9}$ (353.37): C 47.59, H 7.70, N 3.96; found: C 47.55, H 7.68, N 3.93.

Compound $11 \alpha$. Obtained as a yellow oil in quantitative yield starting from $0.023 \mathrm{mmol}$ of $\mathbf{2 6} \alpha$. $[\alpha]_{\mathrm{D}}^{26}=+33.1(c=0.81$ in $\mathrm{MeOH}) ;{ }^{1} \mathrm{H}-\mathrm{NMR}\left(400 \mathrm{MHz}, \mathrm{D}_{2} \mathrm{O}\right): \delta=4.72\left(\mathrm{~d}, J=3.9 \mathrm{~Hz}, 1 \mathrm{H}, \mathrm{H}-\mathrm{1}^{\prime}\right), 3.92(\mathrm{ddd}$, $J=5.4,2.4,2.0 \mathrm{~Hz}, 1 \mathrm{H}, \mathrm{H}-4), 3.73(\mathrm{dd}, J=4.9,3.0 \mathrm{~Hz}, 1 \mathrm{H}, \mathrm{H}-3), 3.67\left(\mathrm{dd}, J=12.2,2.5 \mathrm{~Hz}, 1 \mathrm{H}, \mathrm{Ha}-6^{\prime}\right)$, 3.58-3.46 (m, 6H, H-3' ${ }^{\prime}, \mathrm{Hb}-6^{\prime}, \mathrm{H}-5^{\prime}, \mathrm{H}-6$ and Ha-10), 3.35 (dd, J = 9.7, $\left.3.9 \mathrm{~Hz}, 1 \mathrm{H}, \mathrm{H}-2^{\prime}\right), 3.35-3.32$ (m, $1 \mathrm{H}, \mathrm{Hb}-10), 3.21\left(\mathrm{t}, J=9.7 \mathrm{~Hz}, 1 \mathrm{H}, \mathrm{H}-4^{\prime}\right), 2.82(\mathrm{dd}, J=11.2,1.5 \mathrm{~Hz}, 1 \mathrm{H}, \mathrm{Ha}-5), 2.66$ (ddd, $J=11.7,10.7$, $5.4 \mathrm{~Hz}, 1 \mathrm{H}, \mathrm{Ha}-7), 2.55$ (dd, $J=11.2,5.3 \mathrm{~Hz}, 1 \mathrm{H}, \mathrm{Hb}-5), 2.34$ (q, $J=5.4 \mathrm{~Hz}, 1 \mathrm{H}, \mathrm{H}-2), 2.21$ (dt, J = 11.7, $4.4 \mathrm{~Hz}, 1 \mathrm{H}, \mathrm{Hb}-7), 1.49-1.41$ (m, 2H, H-8), 1.40-1.30 (m, 2H, H-9); ${ }^{13} \mathrm{C}-\mathrm{NMR}\left(100 \mathrm{MHz}, \mathrm{D}_{2} \mathrm{O}\right): \delta=98.0$ $\left(\mathrm{d}, \mathrm{C}-1^{\prime}\right), 79.1(\mathrm{~d}, \mathrm{C}-3), 75.4(\mathrm{~d}, \mathrm{C}-4), 73.1\left(\mathrm{~d}, \mathrm{C}-3^{\prime}\right), 71.8\left(\mathrm{~d}, \mathrm{C}-5^{\prime}\right), 71.7(\mathrm{~d}, \mathrm{C}-2), 71.3\left(\mathrm{~d}, \mathrm{C}-2^{\prime}\right), 69.6\left(\mathrm{~d}, \mathrm{C}-4^{\prime}\right)$, $67.8(\mathrm{t}, \mathrm{C}-10), 61.3(\mathrm{t}, \mathrm{C}-6), 60.5\left(\mathrm{t}, \mathrm{C}-6^{\prime}\right), 58.2(\mathrm{t}, \mathrm{C}-5), 52.4(\mathrm{t}, \mathrm{C}-7), 26.7(\mathrm{t}, \mathrm{C}-8), 23.6(\mathrm{t}, \mathrm{C}-9)$; MS (ESI): $m / z$ calcd (\%) for $\mathrm{C}_{15} \mathrm{H}_{29} \mathrm{NO}_{9}+\mathrm{Na}^{+} 390.18[\mathrm{M}+\mathrm{Na}]^{+}$; found: 390.42 ; elemental analysis calcd $(\%)$ for $\mathrm{C}_{15} \mathrm{H}_{29} \mathrm{NO}_{9}$ (367.39): C 49.04, H 7.96, N 3.81; found: C 49.03, H 7.98, N 3.83. 


\subsection{Synthesis of Compound $9 \beta$}

\subsubsection{Synthesis of 2,3,4,6-Tetra-O-acetyl- $\alpha / \beta$-D-glucopyranose 28 [18]}

To a solution of ethylenediamine (93 mg, $1.54 \mathrm{mmol})$ in $10 \mathrm{~mL}$ of dry THF, acetic acid $(102 \mu \mathrm{L}$, $1.79 \mathrm{mmol}$ ) was slowly added dropwise over $10 \mathrm{~min}$ and the reaction mixture was stirred under nitrogen atmosphere at room temperature for $1 \mathrm{~h}$. Then $27(500 \mathrm{mg}, 1.28 \mathrm{mmol})$ was added and the mixture was stirred for $1 \mathrm{~h}$, until TLC analysis (PE/EtOAc 3:2) showed the disappearance of the starting material $\left(R_{\mathrm{f}}=0.48\right)$ and the formation of a new compound $\left(R_{\mathrm{f}}=0.24\right)$. After washing with $\mathrm{HCl} 0.1 \mathrm{M}(2 \times 2 \mathrm{~mL})$ and $\mathrm{NaHCO}_{3}(2 \times 2 \mathrm{~mL})$, the combined organic layers were dried on $\mathrm{Na}_{2} \mathrm{SO}_{4}$, concentrated at reduced pressure and the crude mixture was purified by FCC (PE/AcOEt 1:1) affording pure $28\left(\alpha / \beta 1: 9 R_{\mathrm{f}}=0.30\right.$, PE/EtOAc 1:1, $383 \mathrm{mg}, 1.100 \mathrm{mmol}, 86 \%$ yield $)$.

${ }^{1} \mathrm{H}-\mathrm{NMR}\left(200 \mathrm{MHz}, \mathrm{CDCl}_{3}\right): \delta=5.62(\mathrm{~d}, J=9.8 \mathrm{~Hz} 1 \mathrm{H}, \mathrm{H}-1 \alpha), 5.55(\mathrm{t}, J=3.8 \mathrm{~Hz}, 1 \mathrm{H}, \mathrm{H}-3 \alpha), 5.36-5.27$ $(\mathrm{m}, 1 \mathrm{H}, \mathrm{H}-3 \beta), 5.14(\mathrm{t}, J=9.6 \mathrm{~Hz}, 1 \mathrm{H} \alpha+1 \mathrm{H} \beta, \mathrm{H}-4), 4.96(\mathrm{dd}, J=9.8,3.4 \mathrm{~Hz}, 1 \mathrm{H} \alpha+1 \mathrm{H} \beta, \mathrm{H}-2)$, $4.80(\mathrm{t}, J=8.1 \mathrm{~Hz}, 1 \mathrm{H}, \mathrm{H}-1 \beta), 4.37-4.12(\mathrm{~m}, 5 \mathrm{H}, \mathrm{H}-6 \alpha, \mathrm{H}-6 \beta, \mathrm{H}-5 \alpha), 3.85-3.77$ (m, 1H, H-5 $\beta$ ), 2.16 (s, $3 \mathrm{H} \alpha+3 \mathrm{H} \beta, \mathrm{OAc}), 2.15$ (s, 3H $\alpha+3 \mathrm{H} \beta, \mathrm{OAc}), 2.09$ (s, 3H $\alpha+3 \mathrm{H} \beta, \mathrm{OAc}), 2.08$ (s, 3H $\alpha+3 \mathrm{H} \beta$, OAc) ppm.

\subsubsection{Synthesis of 2,3,4,6-Tetra-O-acetyl- $\alpha$-D-glucopyranosyltrichloroacetimidate 29 [19]}

A solution of $28(383 \mathrm{mg}, 1.10 \mathrm{mmol})$ in $8 \mathrm{~mL}$ of dry $\mathrm{CH}_{2} \mathrm{Cl}_{2}$ was cooled to $0{ }^{\circ} \mathrm{C}$ and $\mathrm{DBU}(32 \mu \mathrm{L}$, $0.22 \mathrm{mmol})$ and trichloroacetonitrile $(612 \mu \mathrm{L}, 7.70 \mathrm{mmol})$ were added. The reaction mixture was stirred under nitrogen atmosphere at room temperature for $2.5 \mathrm{~h}$, when a TLC analysis (Hex/EtOAc 1:1) showed the disappearance of the starting material $\left(R_{\mathrm{f}}=0.26\right)$ and the formation of a new compound $\left(R_{\mathrm{f}}=0.62\right)$. A saturated $\mathrm{NH}_{4} \mathrm{Cl}$ solution was added and the mixture was transferred to a separating funnel, washing with $\mathrm{CH}_{2} \mathrm{Cl}_{2}$. The organic layer was washed with water $(3 \times 5 \mathrm{~mL})$ and dried over $\mathrm{Na}_{2} \mathrm{SO}_{4}$. After concentration under reduced pressure, the crude was purified by flash column chromatography on silica gel (Hex/EtOAc from 3:1 to 2:1) to afford pure $29\left(R_{\mathrm{f}}=0.25 \mathrm{Hex} / \mathrm{EtOAc} 2: 1\right.$, $461 \mathrm{mg}, 0.939 \mathrm{mmol}, 85 \%$ yield).

${ }^{1} \mathrm{H}-\mathrm{NMR}\left(200 \mathrm{MHz}, \mathrm{CDCl}_{3}\right): \delta=8.69(\mathrm{~s}, 1 \mathrm{H}, \mathrm{NH}), 6.56(\mathrm{~d}, J=3.7 \mathrm{~Hz}, 1 \mathrm{H}, \mathrm{H}-1), 5.57(\mathrm{t}, J=9.8 \mathrm{~Hz}, 1 \mathrm{H}$, H-4), 5.23-5.10 (m, 2H, H-2, H-3), 4.32-4.06 (m, 3H, H-5, H-6 ), 2.08 (s, 3H, OAc), 2.05 (s, 3H, OAc), $2.04(\mathrm{~s}, 3 \mathrm{H}, \mathrm{OAc}), 2.02$ (s, 3H, OAc) ppm.

\subsubsection{Synthesis of Compound 30}

A solution of glucopyranosyl tricholoroacetimidate 29 ( 2 equiv.) and alcohol 15 (1 equiv.) in dry $\mathrm{CH}_{2} \mathrm{Cl}_{2}$, was stirred for $10 \mathrm{~min}$ at room temperature under nitrogen atmosphere in the presence of $3 \AA$ A molecular sieves. After cooling to $0{ }^{\circ} \mathrm{C}$ trimethylsilyl trifluoromethanesulfonate (1.5 equiv.) was added and the mixture was stirred for $1.5 \mathrm{~h}$, letting the temperature to rise. To the reaction mixture $1.8 \mathrm{~mL}$ of triethylamine was added and the mixture was transferred to a separating funnel, washing with $\mathrm{CH}_{2} \mathrm{Cl}_{2}$. The organic layer was washed with $\mathrm{HCl} 1 \mathrm{M}(3 \times 3 \mathrm{~mL}), \mathrm{NaOH} 1 \mathrm{M}(1 \times 3 \mathrm{~mL})$ and brine $(2 \times 3 \mathrm{~mL})$. The combined organic layers were dried over $\mathrm{Na}_{2} \mathrm{SO}_{4}$ and the solvent was removed under reduced pressure. The resulting crude oil was dissolved in pyridine $(3 \mathrm{~mL})$, and acetic anhydride $(2 \mathrm{~mL})$ and DMAP $(30 \mu \mathrm{L})$ were added. The solution was stirred at room temperature overnight, and after concentration under reduced pressure, the crude was purified by flash column chromatography on silica gel (Hex/EtOAc from 1:1 to 1:2) to afford the $\beta$ compound $30\left(R_{\mathrm{f}}=0.516 \mathrm{Hex} /\right.$ EtOAc 1:1, $34 \mathrm{mg}$, $0.044 \mathrm{mmol}, 39 \%$ yield) as colorless oil, contaminated with small amounts of partially deacetylated glucoside (see supplementary information).

Compound 30: $[\alpha]_{\mathrm{D}}^{21}=-15.2\left(c=0.80\right.$ in $\left.\mathrm{CHCl}_{3}\right) ;{ }^{1} \mathrm{H}-\mathrm{NMR}\left(400 \mathrm{MHz}, \mathrm{CDCl}_{3}\right): \delta=7.32-7.24(\mathrm{~m}, 15 \mathrm{H}$, $\mathrm{H}-\mathrm{Ar}), 5.16\left(\mathrm{t}, J=9.5 \mathrm{~Hz}, 1 \mathrm{H}, \mathrm{H}-4^{\prime}\right), 5.05\left(\mathrm{dd}, J=9.8,9.6 \mathrm{~Hz}, 1 \mathrm{H}, \mathrm{H}-2^{\prime}\right), 4.97\left(\mathrm{t}, J=9.6 \mathrm{~Hz}, 1 \mathrm{H}, \mathrm{H}-3^{\prime}\right)$, 4.54-4.43 (m, 7H, H-Bn, H-1' $), 4.23\left(\mathrm{dd}, J=12.2,4.7 \mathrm{~Hz}, 1 \mathrm{H}, \mathrm{Ha}-6^{\prime}\right), 4.09\left(\mathrm{dd}, J=12.2,2.2 \mathrm{~Hz}, 1 \mathrm{H}, \mathrm{Hb}-6^{\prime}\right)$, 
4.00-3.95 (m, 1H, Ha-8), 3.88 (d, $J=5.0 \mathrm{~Hz}, 1 \mathrm{H}, \mathrm{H}-4), 3.81(\mathrm{~d}, J=3.8 \mathrm{~Hz}, 1 \mathrm{H}, \mathrm{H}-3), 3.68-3.58(\mathrm{~m}, 2 \mathrm{H}$, Hb-8, H-5'), 3.56-3.43 (m, 2H, H-6), 3.16 (d, $J=10.7$ Hz, 1H, Ha-5), 3.10-3.04 (m, 1H, Ha-7), 2.77 (q, $J=5.7 \mathrm{~Hz}, 1 \mathrm{H}, \mathrm{H}-2), 2.69-2.61$ (m, 2H, Hb-5, Hb-7), 2.06 (s, 3H, OAc), 2.01 (s, 3H, OAc), 1.99 (s, 3H, OAc), 1.93 (s, 3H, OAc); ${ }^{13} \mathrm{C}-\mathrm{NMR}\left(100 \mathrm{MHz}, \mathrm{CDCl}_{3}\right): \delta=170.6,170.2,169.4,169.3(\mathrm{~s}, 4 \mathrm{C}, \mathrm{C}=\mathrm{O}), 138.3$, 138.2, 138.1 (t, 3C, C-Ar), 128.3, 127.8, 127.7, 127.6, 127.5 (d, 15C, C-Ar), 100.6 (d, C-1'), 84.7 (d, C-4), $81.6(\mathrm{~d}, \mathrm{C}-3), 77.4\left(\mathrm{C}-2^{\prime}\right), 77.1\left(\mathrm{~d}, \mathrm{C}-3^{\prime}\right), 73.2\left(\mathrm{~d}, \mathrm{C}-4^{\prime}\right), 72.8(\mathrm{~d}, \mathrm{C}-2), 71.2,71.1,71.0(\mathrm{~s}, 3 \mathrm{C}, \mathrm{C}-\mathrm{Ar}), 69.4(\mathrm{~d}$, C-5'), 68.9 (t, C-8), $68.4(\mathrm{t}, \mathrm{C}-6), 61.9\left(\mathrm{t}, \mathrm{C}-6^{\prime}\right), 58.4(\mathrm{t}, \mathrm{C}-5), 54.0(\mathrm{t}, \mathrm{C}-7), 20.7,20.6,20.6,20.6\left(\mathrm{q}, 4 \mathrm{C}, \mathrm{CH}_{3}\right)$; IR $\left(\mathrm{CDCl}_{3}\right): v=3031,2945,2866,2360,2331,1755,1497,1454,1375,1231,1171,1039 \mathrm{~cm}^{-1}$. MS (ESI): $m / z$ calcd $(\%)$ for $\mathrm{C}_{42} \mathrm{H}_{51} \mathrm{NO}_{13}+\mathrm{Na}^{+} 800.33[\mathrm{M}+\mathrm{Na}]^{+}$; found: 800.63 .

\subsubsection{Synthesis of Compound $9 \beta$}

To a solution of 30 (43 mg, $0.055 \mathrm{mmol})$ in $10 \mathrm{~mL}$ of methanol, Pd/C $(50 \%, w / w)$ and $0.5 \mathrm{~mL}$ of $\mathrm{HCl}$ $1 \mathrm{M}$ were added under nitrogen atmosphere, then the mixture was stirred under hydrogen atmosphere at room temperature overnight, until an ${ }^{1} \mathrm{H}-\mathrm{NMR}$ control showed the disappearance of the starting material. The mixture was then filtered through Celite ${ }^{\circledR}$ and the solvent was removed under reduced pressure. The crude was purified by flash column chromatography on silica gel $\left(\mathrm{CH}_{2} \mathrm{Cl}_{2} / \mathrm{MeOH} 10: 1\right.$, $R_{\mathrm{f}}=0.585$ ) to afford $12 \mathrm{mg}$, which were dissolved in $10 \mathrm{~mL}$ of methanol, ion exchange resin Ambersep $900-\mathrm{OH}$ was added and the suspension was slowly stirred at room temperature for $16 \mathrm{~h}$. The mixture was filtered through cotton and the solvent was removed under reduced pressure, affording pure $9 \beta$ (8 mg, $0.024 \mathrm{mmol}, 43 \%$ yield) as colorless oil.

Compound 9ß: $[\alpha]_{\mathrm{D}}^{19}=-28.5\left(c=0.67\right.$ in $\left.\mathrm{H}_{2} \mathrm{O}\right) ;{ }^{1} \mathrm{H}-\mathrm{NMR}\left(400 \mathrm{MHz}, \mathrm{D}_{2} \mathrm{O}\right): \delta=4.34(\mathrm{~d}, J=8.0 \mathrm{~Hz}, 1 \mathrm{H}$, H-1'), 4.02-3.98 (m, 1H, H-4), 3.92 (ddd, $J=11.0,6.4,4.4$ Hz, 1H, Ha-8), 3.82-3.81 (m, 1H, H-3), 3.77 (d, $\left.J=2 \mathrm{~Hz}, 1 \mathrm{H}, \mathrm{Ha}-6^{\prime}\right), 3.68-3.56$ (m, 4H, Hb-8, H-6, Hb-6'), 3.38-3.21 (m, 3H, H-3', H-4', , H-5'), 3.15 (t, $\left.J=8.4 \mathrm{~Hz}, 1 \mathrm{H}, \mathrm{H}-2^{\prime}\right), 3.05-2.97$ (m, 2H, Ha-7, Ha-5), $2.74(\mathrm{dd}, J=11.4,5.7 \mathrm{~Hz}, 1 \mathrm{H}, \mathrm{Hb}-5), 2.61-2.53$ (m, 2H, Hb-7, H-2); ${ }^{13} \mathrm{C}-\mathrm{NMR}\left(100 \mathrm{MHz}, \mathrm{D}_{2} \mathrm{O}\right): \delta=102.32\left(\mathrm{~d}, \mathrm{C}-1^{\prime}\right), 79.11(\mathrm{~d}, \mathrm{C}-3), 76.06\left(\mathrm{~d}, \mathrm{C}-3^{\prime}\right), 75.79(\mathrm{~d}$, C-5'), $75.62(\mathrm{~d}, \mathrm{C}-4), 73.20\left(\mathrm{~d}, \mathrm{C}-2^{\prime}\right), 72.01(\mathrm{~d}, \mathrm{C}-2), 69.78\left(\mathrm{~d}, \mathrm{C}-4^{\prime}\right), 67.88(\mathrm{t}, \mathrm{C}-8), 61.19(\mathrm{t}, \mathrm{C}-6), 60.88(\mathrm{t}$, C-6 $\left.6^{\prime}\right), 58.94$ (t, C-5), 54.04 (t, C-7); MS (ESI): $m / z$ calcd (\%) for $\mathrm{C}_{13} \mathrm{H}_{25} \mathrm{NO}_{9}+\mathrm{Na}^{+} 362.15$ [M + Na] ; found: 362.47; elemental analysis calcd (\%) for $\mathrm{C}_{13} \mathrm{H}_{25} \mathrm{NO}_{9}$ (339.34): C 46.01, $\mathrm{H}$ 7.43, N 4.13; found: C 46.67, H 7.45, N 3.20.

\subsection{Biological Evaluation of Compounds $\mathbf{8}, \mathbf{9} \alpha, \mathbf{9} \beta, \mathbf{1 0} \alpha, \mathbf{1 1} \alpha$ and the $\alpha / \beta$ Mixture of $\mathbf{9 , 1 0}$ and $\mathbf{1 1}$}

Compounds were tested for their inhibitory activity against insect trehalase of midge larvae of C. riparius, a good model for biochemical studies [20], and porcine kidney trehalase (purchased from Sigma-Aldrich, St. Louis, MO, USA) as the mammalian counterpart.

Trehalase activity was measured through a coupled assay with glucose-6-phosphate dehydrogenase and hexokinase according to Wegener at al. [21] To examine the potential of each compound as a trehalase inhibitor, dose-response curves were established to determine the $\mathrm{IC}_{50}$ values. Experiments were performed at a fixed substrate concentration close to the $K_{\mathrm{m}}$ value ( $0.5 \mathrm{mM}$ for C. riparius and $2.5 \mathrm{mM}$ for porcine trehalase), in the presence of increasing inhibitor concentrations.Initial rates as a function of inhibitor concentration were fitted to the following equation:

$$
\frac{v_{i}}{v}=\frac{1}{1+\left(\frac{[I]}{I C_{50}}\right)^{n}}
$$

where $v_{i}$ and $v$ are the initial rate in the presence and in the absence of inhibitor, respectively, $[I]$ is the inhibitor concentration, $\mathrm{IC}_{50}$ is the inhibitor concentration producing half-maximal inhibition, and $n$ is the Hill coefficient.

Kinetic experiments were performed using $C$. riparius trehalase, measuring enzymatic activity at different trehalose concentrations from 0.05 to $20 \mathrm{mM}$ in the presence of fixed inhibitor concentrations (5-10 $\mu \mathrm{M}$ for the compound $9 \alpha$ and $0.5-1 \mu \mathrm{M}$ for the compound $9 \beta$ ). Kinetic parameters were calculated 
using a multiparameter, iterative, non-linear regression program based on the Marquardt-Levenberg algorithm (Sigma Plot, Jandel, CA, USA). Data are given \pm S.D. of three independent experiments.

All enzyme assays were performed in triplicates at $30^{\circ} \mathrm{C}$ by using sample volumes varying from 5 to $20 \mu \mathrm{L}$ in $1 \mathrm{~mL}$ test and using a Cary3 UV/Vis Spectrophotometer. Enzyme activities were analyzed by Cary Win UV application software for Windows XP.

\section{Conclusions}

In conclusion, the synthesis of new pseudodisaccharide mimics 8, 9, 10 and $\mathbf{1 1}$, bearing a glucosyl moiety and a pyrrolizidine or pyrrolidine portion, was undertaken and their biological activity as insect trehalase inhibitors was evaluated. Inversion of configuration at C-6 in compound 8 (with respect to previously synthesized compound 4) resulted in a decrease of potency and selectivity towards the insect trehalase, showing the key role played by the stereochemical configuration at C-6 of the pyrrolizidine nucleus.

Moreover, a simple synthetic strategy was developed to obtain new pseudodisaccharide inhibitors with a pyrrolidine core, demonstrating the pivotal function played by the distance between the glucosyl and the iminosugar pyrrolidine moiety in these flexible inhibitors. Indeed, among a series of new compounds 9-11, only compounds 9 (with a two-carbon atom linker) maintained their inhibitory activity, while compounds $\mathbf{1 0}$ and $\mathbf{1 1}$ were completely inactive. In particular, the stereoselective synthesis of compound $9 \beta$ allowed the identification of a new and selective insect trehalase inhibitor with $\mathrm{IC}_{50}$ in the low micromolar range $\left(\mathrm{IC}_{50}=0.78 \mu \mathrm{M}\right)$.

Supplementary Materials: The following are available online at www.mdpi.com/link, ${ }^{1} \mathrm{H}$ and ${ }^{13} \mathrm{C}-\mathrm{NMR}$ spectra of new compounds and Figures S1-S4. Inhibition kinetics of insect trehalase in the presence of compounds $9 \alpha$ and $9 \beta$ and dose-response curves of compounds $8,9 \alpha, \beta, 9 \alpha$ and $9 \beta$ for insect and porcine trehalase.

Acknowledgments: Ente Cassa di Risparmio di Firenze is acknowledged for a fellowship to GD (grant No. 2014/0303). CM thanks Accademia dei Lincei/Fondazione Donegani for a fellowship.

Author Contributions: F.C. and C.M. planned the synthetic strategy and wrote the manuscript. G.D., X.F. and C.V. made the syntheses, C.M. contributed to the N.M.R. characterization of the new compounds, M.F., P.F. and P.P. performed the biological data and contributed to the writing of the manuscript.

Conflicts of Interest: The authors declare no conflict of interest. The founding sponsors had no role in the design of the study; in the collection, analyses, or interpretation of data; in the writing of the manuscript, and in the decision to publish the results.

\section{References}

1. Elbein, A.D.; Pan, Y.T.; Pastuszak, I.; Carroll, D. New insights on trehalose: A multifunctional molecule. Glycobiology 2003, 13, 17R-27R. [CrossRef] [PubMed]

2. Defaye, J.; Driguez, H.; Henrissat, B.; Bar-Guilloux, E. Stereochemistry of the hydrolysis of $\alpha, \alpha$-trehalose by trehalase, determined by using a labelled substrate. Carbohydr. Res. 1983, 124, 265-273. [CrossRef]

3. Bini, D.; Cardona, F.; Gabrielli, L.; Russo, L.; Cipolla, L. Trehalose mimetics as inhibitors of trehalose processing enzymes. In Carbohydrate Chemis Try: Volume 37; Rauter, A.P., Lindhorst, T., Eds.; Royal Society of Chemistry: Cambridge, UK, 2012; pp. 259-302, ISBN 978-1-84973-154-6.

4. Gibson, R.P.; Gloster, T.M.; Roberts, S.; Warren, R.A.; de Gracia, S.; García, Á.; Chiara, J.L.; Davies, G.J. Molecular basis for trehalase inhibition revealed by the structure of trehalase in complex with potent inhibitors. Angew. Chem. Int. Ed. 2007, 46, 4115-4119. [CrossRef] [PubMed]

5. Cardona, F.; Parmeggiani, C.; Faggi, E.; Bonaccini, C.; Gratteri, P.; Sim, L.; Gloster, T.M.; Roberts, S.; Davies, G.J.; Rose, D.R.; et al. Total syntheses of casuarine and its 6-O- $\alpha$-glucoside: Complementary inhibition towards glycoside hydrolases of families GH31 and GH37. Chem. Eur. J. 2009, 15, 1627-1636. [CrossRef] [PubMed]

6. Cardona, F.; Goti, A.; Parmeggiani, C.; Parenti, P.; Forcella, M.; Fusi, P.; Cipolla, L.; Roberts, S.M.; Davies, G.J.; Gloster, T.M. Casuarine-6-O- $\alpha$-D-glucoside and its analogues are tight binding inhibitors of insect and bacterial trehalases. Chem. Commun. 2010, 46, 2629-2631. [CrossRef] 
7. D'Adamio, G.; Sgambato, A.; Forcella, M.; Caccia, S.; Parmeggiani, C.; Casartelli, M.; Parenti, P.; Bini, D.; Cipolla, L.; Fusi, P.; et al. New synthetic and biological evaluation of uniflorine A derivatives: Towards specific insect trehalase inhibitors. Org. Biomol. Chem. 2015, 13, 886-892. [CrossRef] [PubMed]

8. Bini, D.; Forcella, M.; Cipolla, L.; Fusi, P.; Matassini, C.; Cardona, F. Synthesis of novel iminosugar-based trehalase inhibitors by Cross-Metathesis reactions. Eur. J. Org. Chem. 2011, 3995-4000. [CrossRef]

9. Bini, D.; Cardona, F.; Forcella, M.; Parmeggiani, C.; Parenti, P.; Nicotra, F.; Cipolla, L. Synthesis and biological evaluation of nojirimycin- and pyrrolidine-based trehalase inhibitors. Beilstein J. Org. Chem. 2012, 8, 514-521. [CrossRef] [PubMed]

10. Cardona, F.; Faggi, E.; Liguori, F.; Cacciarini, M.; Goti, A. Total syntheses of hyacinthacine $\mathrm{A}_{2}$ and 7-deoxycasuarine by cycloaddition to a carbohydrate derived nitrone. Tetrahedron Lett. 2003, 44, 2315-2318. [CrossRef]

11. Overkleeft, H.S.; van Wiltenburg, J.; Pandit, U.K. A facile transformation of sugar lactones to azasugars. Tetrahedron 1994, 50, 4215-4224. [CrossRef]

12. Zhou, X.; Liu, W.-J.; Ye, J.-L.; Huang, P.-Q. A versatile approach to pyrrolidine azasugars and homoazasugars based on a highly diastereoselective reductive benzyloxymethylation of protected tartarimide. Tetrahedron 2007, 63, 6346-6357. [CrossRef]

13. D'Adamio, G.; Matassini, C.; Parmeggiani, C.; Catarzi, S.; Morrone, A.; Goti, A.; Paoli, P.; Cardona, F. Evidence for a Multivalent Effect in Inhibition of Sulfatases Involved in Lysosomal Storage Disorders (LSDs). RSC Adv. 2016, 6, 64847-64851. [CrossRef]

14. Damager, I.; Olsen, C.E.; Møller, B.L.; Motawia, M.S. Chemical synthesis of $6^{\prime \prime \prime}$ - $\alpha$-maltotriosyl-maltohexaose as substrate for enzymes in starch biosynthesis and degradation. Carbohydr. Res. 1999, 320, 19-30. [CrossRef]

15. Stick, R.V.; Williams, S.J. Formation of the glycosidic linkage. In Carbohydrates the Essential Molecules of Life, 2nd ed.; Stick, R.V., Williams, S.J., Eds.; Elsevier: Amsterdam, The Netherlands, 2009; pp. 1133-1202, ISBN 978-0-240-52118-3.

16. Wang, W.; Kong, F. New Synthetic Methodology for Regio- and Stereoselective Synthesis of Oligosaccharides via Sugar Ortho Ester Intermediates. J. Org. Chem. 1998, 63, 5744-5745. [CrossRef] [PubMed]

17. Sun, Q.; Yang, Q.; Gong, S.; Fu, Q.; Xiao, Q. Synthesis and enzymatic evaluation of phosphoramidon and its $\beta$ anomer: Anomerization of $\alpha$-L-rhamnose triacetate upon phosphitylation. Bioorg. Med. Chem. 2013, 21, 6778-6787. [CrossRef] [PubMed]

18. Fernandez-Lorente, G.; Palomo, J.M.; Cocca, J.; Mateo, C.; Moro, P.; Terreni, M.; Fernandez-Lafuenteb, R.; Guisanb, J.M. Regio-selective deprotection of peracetylated sugars via lipase hydrolysis. Tetrahedron 2003, 59, 5705-5711. [CrossRef]

19. Koketsu, M.; Kuwahara, M. First Synthesis of a Trisaccharide of Glycosylkaemferide: A Resistance Factor in Carnations. Synth. Commun. 2004, 34, 239-245. [CrossRef]

20. Forcella, M.; Mozzi, A.; Bigi, A.; Parenti, P.; Fusi, P. Molecular cloning of soluble trehalase from Chironomus riparius larvae, its heterologous expression in Escherichia coli and bioinformatic analysis. Arch. Insect Biochem. Physiol. 2012, 81, 77-89. [CrossRef] [PubMed]

21. Wegener, G.; Tschiedel, V.; Schlöder, P.; Ando, O.J. The toxic and lethal effects of the trehalase inhibitor trehazolin in locusts are caused by hypoglycaemia. J. Exp. Biol. 2003, 206, 1233-1240. [CrossRef] [PubMed]

Sample Availability: Samples of the compounds are not available from the authors. 